\title{
Simulating Wheat Production Potential Under Near-future Climate Change in Arid Regions of Northeast Iran
}

Seyed Farhad Saberali ( $\nabla$ sf.saberali@yahoo.com )

University of Torbat-e-Jam https://orcid.org/0000-0002-1868-1313

Zahra Shirmohammadi-Aliakbarkhani

University of Torbat-e-Jam

Hossein Nastari Nasrabadi

University of Torbat-e-Jam

\section{Research Article}

Keywords: Climate projections, DSSAT, evapotranspiration, internal renewable water resources, Irrigation efficiency

Posted Date: January 10th, 2022

DOI: https://doi.org/10.21203/rs.3.rs-1219301/v1

License: () (i) This work is licensed under a Creative Commons Attribution 4.0 International License. Read Full License 


\section{Abstract}

Water scarcity is the key challenge in arid regions, which exacerbates under climate change (CC) and must be considered to assess the impacts of CC on cropping systems. A climate-crop modelling approach was employed by using the CSM-CERES-Wheat model in some arid regions of northeast Iran to project the effects of CC on irrigated wheat production. Current climate data for 1990-2019 and climate projections of three climate models for 2021-2050 under RCP4.5 and RCP8.5 emission scenarios were used to run the crop model. Two irrigation scenarios with different irrigation efficiencies were also simulated to investigate the impacts of water scarcity associated with changing climate and irrigation management on wheat productivity. Results indicated that mean temperature is projected to increase at the rates of $1.74-2.73^{\circ} \mathrm{C}$ during the reproductive growth period of winter wheat over the study areas. The precipitation projections also indicated that the precipitation rates would decrease over most of the wheat-growing period. The length of the vegetative growth period will extend in some regions and shorten in others under the near future climate. However, the grain filling duration will reduce by about $2-4$ days across all regions. The mean seasonal PET is expected to decrease by about $11 \mathrm{~mm}$ from 2021 to 2050 over the study areas. A mean overall reduction in winter wheat yield due to future climate conditions would be about $12.3 \%$ across the study areas. However, an increase of $15-30 \%$ in the irrigation efficiency will be able to offset yield reductions associated with limited water supply under future climate scenarios. The results suggest that $\mathrm{CC}$ will exacerbate limited irrigation water availability, so implementing high-efficiency irrigation systems should be a priority to adapt to climate change in an arid cropping system.

\section{Introduction}

Arid and semi-arid regions constitute almost $40 \%$ of the world's land surfaces and are home to over 2 billion people, i.e., $35 \%$ of the world's population (ElBeltagy and Madkour 2012). The arid zone is characterized by excessive heat, low and variable precipitation, and intensive evaporation (Salem 1989). There is an overall deficit in the annual water balance of arid regions, and the amount of that deficit determines the degree of aridity. CC is going to have a negative impact on dryland ecosystems, where cover $98 \%$ of Iran's lands and constitute the majority of cropland (Mesgaran et al. 2017).

The CC refers to any long-term change in the typical weather patterns such as temperature and rainfall in a region (IPCC 2014). The CC has challenged food security by affecting agricultural crop production and become a critical global and national issue. Crop production faces the challenge of changing climate, particularly due to decreasing available water resources and rising temperatures (Abbaspour et al. 2009; Karandish et al. 2017). The impact of CC in the 21st century will very likely result in global warming and more frequent heatwaves, and also changes the distribution and intensity of precipitation which could increase the local impacts of water scarcity, as well as the frequency of floods (El-Beltagy and Madkour 2012; Ashraf Vaghefi et al. 2019). The temperature in the Middle East was projected to increase by 2.5 to $4 \mathrm{C}$ by the end of the 21 st century. Iran, Iraq, Turkey, and Afghanistan are projected to experience the highest temperature increases across the Middle East in the future (Bucchignani et al. 2018). The CC also is expected to have strong impacts on hydrological processes such as precipitation and evapotranspiration across different regions and consequently on the spatial and temporal distributions of water resources. The $\mathrm{CC}$ analysis showed that the maximum decrease in the water supply would occur in the West Asia and North Africa region (El-Beltagy and Madkour 2012). The CC is forecast to exacerbate already existing water scarcity challenges in arid and semi-arid regions of Iran (Abbaspour et al. 2009; Zamanirad et al. 2018). About $90 \%$ of Iran's agricultural products are harvested from irrigated lands (Agricultural Statistical Yearbook 2018), where crop water demand cannot be met by rainwater, and irrigation is used to supplement the crop's water needs. In most regions of Iran, unrestrained irrigation use and climate change over the past decades aggravated groundwater overdraft, particularly in arid and semi-arid regions (Dalin et al. 2017; Madani et al. 2016).

In recent decades, the impact of CC on irrigated crop production has most likely been due to rising temperatures and carbon dioxide levels since reduced precipitation and water shortage during the growing season have been offset by more irrigation. Therefore, the effect of drought stress associated with CC on crop production has been diminished at the expense of overexploitation of groundwater resources (non-renewable water resources), which has been formed from the water storage in the subsurface over the past years (Madani et al. 2016). Today, an adequate irrigation water supply is a serious challenge for cropping systems in arid areas, and water availability completely depends on the renewable water supply capacity because of groundwater storage depletion. Understanding the magnitude and extent of the CC impacts on water availability and cropping systems is critical to design policies that outline effective adaptation strategies. Crop simulation models are useful tools to aid decision-makers in projecting changes in crop production and defining adaptation strategies under changing environments. Crop models have become increasingly prominent in the $\mathrm{CC}$ research owing to their utility to simulate the interactions among genetics, weather, and management (e.g. irrigation management) factors (Jones et al. 2003; Nouri et al. 2017).

Wheat (Triticum aestivum L.) plays an important role in maintaining global and national food security and is the most widely grown crop in arid and semi-arid cropping zones of Iran. Irrigated wheat accounts for $33 \%$ of the total irrigated land in Iran, and groundwater is the main source of irrigation water supply (Agricultural Statistical Yearbook 2018). Numerous studies have been conducted using crop models to project the effects of climate change on wheat productivity around the world (Sommer et al. 2013; Dettori et al. 2017; Kothari et al. 2019; Eyni-Nargeseh et al. 2019), but most of these studies have not considered the effect of irrigation water availability associated with CC on irrigated cropping systems. Assessing the effects of CC and future water availability on irrigated wheat production is essential for studying the plant responses, finding adaptation strategies, and minimizing risks. From this perspective, the objectives of this study were to: (1) understand patterns of change in climatic variables during the winter wheat growing season for the near future (20212050) in northeast Iran; (2) quantify CC impact on future water availability; (3) estimate the CC impacts on wheat phenology and production during the near future (2021-2050) relative to the baseline (1990-2019); (4) identify the optimum irrigation efficiency as an adaptation strategy to climate change.

\section{Materials And Methods}

Study area

The total cultivated area has been fluctuating around 12 million hectares over the past 25 years in Iran, and Khorasan Razavi Province, with $7 \%$ of the total cultivated area, occupies the third position of crop production in Iran. The province is located in the northeast of Iran, between $33^{\circ}-38^{\circ} \mathrm{N}$ latitude and $56^{\circ}-62^{\circ} \mathrm{E}$ 
longitude, with an altitude range from $235 \mathrm{~m}$ to $1460 \mathrm{~m}$ (Fig. 1). The province encompasses approximately $117,200 \mathrm{~km}^{2}$ and covers about $10 \%$ of the total irrigated wheat production areas in Iran (Agricultural Statistical Yearbook 2018). The climate is a Mediterranean type with an annual daily mean temperature of $15.9^{\circ} \mathrm{C}$ and annual precipitation of $250 \mathrm{~mm}$. Four counties within the province were selected as the study area to evaluate climate change impacts. The selected sites, Neyshabour, Mashhad, Sabzevar, and Torbat-e Jam, constitute about $40 \%$ of the total irrigated wheat production in the province. These regions were designated as critical groundwater areas. The climate of the study areas is classified as arid, according to Thornthwaite (1948).

Crop model

The Decision Support System for Agrotechnology Transfer (DSSAT) is a software package that comprises crop simulation models to simulate daily growth and phenological development in response to soil properties, weather conditions, and management practices for over 42 crops, as well as tools to facilitate effective use of the models (Hoogenboom et al. 2019). The CSM-CERES-Wheat model is one of the main physiologically-based crop models in the DSSAT software package, simulates the growth and development, productivity, water, and nitrogen balances for wheat, based on cultivar-specific coefficients (Jones et al. 2003). The CSM-CERES-Wheat model has been widely applied for the assessment of potential impacts related to CC on wheat production around the world (Mishra et al. 2013; Dettori et al. 2017; Nouri et al. 2017). The model uses a radiation use efficiency based approach to calculate dry matter production, and sub-optimal water and nitrogen supply can reduce the potential dry matter production. The biomass is partitioned into different plant components according to a set of partitioning coefficients in each growth stage. Crop development is a function of accumulated degree-days, photoperiod, and verbalization requirements. A detailed introduction of the DSSAT cropping system models was documented by Jones et al. (2003).

Model input data

The CSM-CERES-Wheat model simulates wheat growth and development at a daily time step based on daily weather conditions, soil characteristics, cultivarspecific coefficients, and management practices (Hoogenboom et al. 2019). The minimum weather data requirements are the daily minimum and maximum air temperatures, solar radiation, and rainfall. WeatherMan utility program was used to prepare a weather data file. Available historical meteorological data over a 27-30 year period was obtained from the provincial meteorological office for each study site, including Mashhad (1990-2019), Neyshabour (1992-2019), Sabzevar (1990-2019), and Torbat-e Jam (1993-2019). The locations of the selected weather stations, climate type, and historical observations periods are shown in Table 1.

Future climate data were obtained from the General Circulation Models (GCMs) for different study areas over the near future period (2021-2050). The Fifth Assessment Report (AR5) of the Intergovernmental Panel on Climate Change (IPCC) has developed climate projections based on the levels of greenhouse gas concentrations, and we selected two Representative Concentration Pathways (RCP) 4.5 and 8.5 (IPCC, 2014). The RCP 4.5 pathway is the intermediate stabilization scenario which can only be achieved if proper adaptation policies are implemented. The RCP 8.5 pathway represents a future with a high greenhouse gas concentration in the absence of climate mitigation policies. Therefore, the CC impact assessment was carried out under mitigated (RCP 4.5) and unmitigated (RCP 8.5) emission scenarios. MarkSimGCM was employed to downscale coarse-scale GCMs outputs to a $0.5^{\circ} \times 0.5^{\circ}$ latitude/longitude grid resolution using stochastic downscaling and climate typing techniques (Jones and Thornton 2013). The model collects the baseline period climate data (1961-1990) from the World-Clim database. This application was originally designed for use with DSSAT, and its current version can generate future daily weather data based on 17 GCMs under different RCPs (Jones and Thornton, 2013). It is recommended to use multiple GCMs to generate a range of possible climate futures for considering uncertainty in climate model projections (Hawkins and Sutton, 2011). Doulabian et al. (2020) evaluated the accuracy of the 25 GCMs model for the air temperature and precipitation projections over the historical period (1981-2000) around Iran. Regarding their evaluation, the MIROC5, GFDL-ESM2G, and HadGEM2-ES models were selected as high accuracy models for projecting the climate data in the study areas. The $\mathrm{CO}_{2}$ level was varied using the "Environmental Modifications" option in the crop management data tool of DSSAT. The $\mathrm{CO}_{2}$ concentration in the mid baseline period (2005s) was set at 377 ppm (https://www.climate.gov/news-features/understanding-climate/climate-change-atmospheric-carbon-dioxide) for baseline simulations and in the mid-future period (2035) were considered to be 448 and 468 ppm for simulations under RCP 4.5 and RCP 8.5, respectively (IPCC 2014).

Soil inputs data were derived from the Harmonized World Soil Database (HWSD), the most up-to-date world soil map (FAO/IIASA/ISRIC/ISS-CAS/JRC, 2012), and also some available soil survey reports. The HWSD V.1.2 is a 30 arc-second raster database with over 15000 different soil mapping units that combine existing regional and national updates of soil information worldwide, and is available at: http://www.fao.org/soils-portal/soil-survey/soil-maps-anddatabases/harmonized-world-soil-database-v12/en/. The soils of the study areas are classified as Calcisols, Leptosols, Regosols and Solonchaks based on the world reference base for soil resources (IUSS Working Group WRB 2006). Local soil survey reports showed that loam, clay loam, and sandy clay loam are the major soil texture type in the wheat production areas. The different soil types were used in simulations to consider the effect of soil texture on wheat production changes under CC impact.

Data on management practices were obtained through field surveys and consultation with local agricultural experts at the Iranian Ministry of Agriculture. A summary of the main agronomic management information used to initialize the CSM-CERES-Wheat simulations is reported in Table 2. Two irrigation scenarios with different irrigation efficiencies were also simulated to investigate the impact of future irrigation water shortage on wheat productivity and analyze the potential of improved irrigation efficiency to cover it up. Based on the field survey data, the current farmer-applied irrigation amount for irrigated wheat is on average about $5300 \mathrm{~m}^{3} \mathrm{ha}^{-1}$ in Mashhad and Neyshabour, and $6500 \mathrm{~m}^{3} \mathrm{ha}^{-1}$ in Sabzevar and Torbat-e jam. The aggregate irrigation efficiency has been about $40 \%$ over the recent decade in the study areas. The current farmer-applied irrigation amounts were used for the baseline simulations and also future simulations when no further irrigation shortage is expected. In the deficit irrigation scenarios, the rates of decrease in internal renewable water resource (IRWR) under both climate scenarios compared to the baseline period were used as a criterion for the amount of irrigation water shortage in future climate. As the most important adaptation strategy utilized by the farmers in response to the water deficit in recent decades, we also evaluated the effectiveness of improving irrigation efficiency in adapting to water scarcity associated with CC. 
Modeling

In Iran over the last two decades, most of the stored groundwater was depleted by wells and Qantas (gently sloping underground tunnels) and used for irrigation (Dalin et al. 2017, Mirzaei et al. 2019). So, we supposed that future irrigation water supply would greatly depend on the annual internal renewable water resources in each region. The IRWR is a function of the precipitation and evapotranspiration, which are affected by climate change phenomena. Thus, the natural logarithmic transformation of the observed IRWR was depicted against the ratio of mean annual precipitation $(P)$ to mean annual reference evapotranspiration ( $\left(\mathrm{ET}_{\mathrm{o}}\right.$ ) for 101 countries around the world. The different functions were fitted to the data, and the best fitting function with the highest coefficient of determination $\left(\mathrm{R}^{2}\right)$ and lowest root mean square error (RMSE) was used to estimate changes in IRWR for each region. The selected countries have higher annual rates of $\mathrm{ET}_{0}$ than $\mathrm{P}$, similar to the study area. The data for the observed IRWR, $\mathrm{P}$, and estimated ET。 of different countries over $1960-2015$ was obtained from the FAO AQUASTAT database (http://www.fao.org/nr/water/aquastat/data/query/index.html?lang=en, and http://www.fao.org/aquastat/en/climate-info-tool/). In the study areas, the FAO Penman-Monteith method (Allen et al. 1998) was used to estimate ET。 over the baseline period in each region. Because of the limited weather data under future climate scenarios, Hargreaves-Samani or Jensen-Haise method was used as a reliable alternative method to estimate $\mathrm{ET}_{0}$ (Shirmohammadi-aliakbarkhani and Saberali 2020). Finally, the calibrated model was used to simulate wheat growth under different climate change scenarios, soil textures, irrigation water availability, and irrigation efficiency in arid regions. We calibrated and evaluated the model using field data from the published and unpublished studies on wheat response to planting dates and irrigation regimes across the study areas (Jafarnejad and Sharif-Alhosseini 2010; Saberali et al. 2019). Cultivar coefficients of the winter wheat (Pishtaz) were estimated using these data, as the parameters were adjusted manually to get a good match between measured and simulated crop development and growth traits. The coefficients for phenotypic and growth characterization of this cultivar are reported in Table 3. The model performance during the calibration and evaluation period was assessed by three different statistics, including root mean square error (RMSE), normalized root mean square error (nRMSE), and means bias error (MBE) (Jamieson et al. 1991; Nouri et al. 2017). The nRMSE values lower than 20\% indicate a good model performance, while the RMSE and MBE values close to zero indicate better performance.

\begin{tabular}{|llllll|}
\hline \multicolumn{4}{|l|}{ Table 1 Weather station locations and period of meteorological observations. } \\
\hline Station & Latitude & Longitude & Elevation $(\mathrm{m})$ & Observation period & Climate \\
\hline Mashhad & $36 \square 14 \square \mathrm{N}$ & $59 \square 38 \square \mathrm{E}$ & 999 & $1990-2019$ & Arid \\
\hline Neyshabour & $36 \square 16 \square \mathrm{N}$ & $58 \square 48 \square \mathrm{E}$ & 1213 & $1992-2019$ & Arid \\
\hline Sabzevar & $36 \square 12 \square \mathrm{N}$ & $57 \square 39 \square \mathrm{E}$ & 962 & $1990-2019$ & Arid \\
\hline Torbat-e Jam & $35 \square 16 \square \mathrm{N}$ & $60835 \square \mathrm{E}$ & 950 & $1993-2019$ & Arid \\
\hline
\end{tabular}

Table 2 Details of the main crop management information used to initialize the CSM-CERES-Wheat crop model for the different climate scenarios.

\begin{tabular}{|c|c|c|c|}
\hline \multirow[t]{2}{*}{ Features } & \multirow{2}{*}{$\begin{array}{l}\text { Baseline period } \\
(1990-2019)\end{array}$} & \multicolumn{2}{|c|}{ Future climate change scenarios (2021-2050) } \\
\hline & & $\mathrm{RCP} 4.5$ & RCP8.5 \\
\hline Planting date & 16 October & & \\
\hline Plant density & 300 plant $\mathrm{m}^{-2}$ & & \\
\hline Planting depth & $5 \mathrm{~cm}$ & & \\
\hline \multirow[t]{2}{*}{ Irrigation amount } & $\star 5300 \mathrm{~m}^{3} \mathrm{ha}^{-1}$ & 5300 and $4587 \mathrm{~m}^{3} \mathrm{ha}^{-1}$ & 5300 and $3826 \mathrm{~m}^{3} \mathrm{ha}^{-1}$ \\
\hline & $+6500 \mathrm{~m}^{3} \mathrm{ha}^{-1}$ & 6500 and $5620 \mathrm{~m}^{3} \mathrm{ha}^{-1}$ & 6500 and $4930 \mathrm{~m}^{3} \mathrm{ha}^{-1}$ \\
\hline Irrigation efficiency & $40 \%$ & 40,55 and $70 \%$ & \\
\hline Nitrogen fertilizer rate & $150 \mathrm{~kg} \mathrm{ha}^{-1}$ & & \\
\hline $\mathrm{Co}_{2}$ concentration & 377 ppm & 448 ppm & 468 ppm \\
\hline
\end{tabular}

* Amount of irrigation water application in Mashhad and Neyshabour

† Amount of irrigation water application in Sabzevar and Torbat-e Jam 
Table 3 Genetic coefficients of the Pishtaz wheat cultivar for the CSM-CERES-Wheat model.

\begin{tabular}{llc} 
Coefficients & Definition & Value \\
\hline P1V & Days at the optimum vernalizing temperature required to complete vernalization & 10 \\
\hline P1D & Percentage reduction in development rate in a photoperiod shorter than the threshold $(10$ hours $)$ & 60 \\
\hline P5 & Duration of the grain-filling phase $\left({ }^{\circ} \mathrm{C}\right.$ d $)$ & 600 \\
\hline G1 & Kernel number per unit canopy weight at anthesis (kernels g- 1$)$ & 20 \\
\hline G2 & Standard kernel size under optimum conditions $(\mathrm{mg})$ & 30 \\
\hline G3 & Standard, non-stressed mature tiller weight (including grain $)(\mathrm{g}$ dry weight $)$ & 2 \\
\hline PHINT & The interval between successive leaf tip appearances $\left({ }^{\circ} \mathrm{C}\right.$ d) & 95
\end{tabular}




\begin{tabular}{|c|c|c|c|c|c|c|c|}
\hline Assessment phase & Variable & Year & Observed & Simulated & RMSE & nRMSE (\%) & Mean bias error (MBE) \\
\hline \multirow[t]{15}{*}{ Calibration } & \multirow{3}{*}{$\begin{array}{l}\text { Anthesis date } \\
\text { (Days after planting) }\end{array}$} & $2004-2005$ & 200 & 203 & \multirow[t]{3}{*}{2.10} & \multirow[t]{3}{*}{1.10} & \multirow[t]{3}{*}{-1.7} \\
\hline & & $2010-2011$ & 190 & 192 & & & \\
\hline & & 2015-2016 & 188 & 188 & & & \\
\hline & \multirow{3}{*}{$\begin{array}{l}\text { Maturity date } \\
\text { (Days after planting) }\end{array}$} & 2004-2005 & 233 & 237 & \multirow[t]{3}{*}{2.65} & \multirow[t]{3}{*}{1.18} & \multirow[t]{3}{*}{-2.33} \\
\hline & & $2010-2011$ & 221 & 223 & & & \\
\hline & & 2015-2016 & 217 & 218 & & & \\
\hline & \multirow[t]{3}{*}{ LAl } & $2004-2005$ & 6.1 & 6.5 & \multirow[t]{3}{*}{0.48} & \multirow[t]{3}{*}{7.40} & \multirow[t]{3}{*}{-0.47} \\
\hline & & 2010-2011 & 6.4 & 6.8 & & & \\
\hline & & 2015-2016 & 6.8 & 7.4 & & & \\
\hline & \multirow[t]{3}{*}{ Biomass (kg ha $\left.{ }^{-1}\right)$} & 2004-2005 & 16040 & 14932 & \multirow[t]{3}{*}{777.7} & \multirow[t]{3}{*}{4.72} & \multirow[t]{3}{*}{10.33} \\
\hline & & $2010-2011$ & 16231 & 16712 & & & \\
\hline & & 2015-2016 & 17103 & 17699 & & & \\
\hline & \multirow[t]{3}{*}{ Yield $\left(\mathrm{kg} \mathrm{ha}^{-1}\right)$} & $2004-2005$ & 6425 & 6001 & \multirow[t]{3}{*}{375.6} & \multirow[t]{3}{*}{5.61} & \multirow[t]{3}{*}{-85} \\
\hline & & 2010-2011 & 6650 & 6909 & & & \\
\hline & & 2015-2016 & 7012 & 7432 & & & \\
\hline \multicolumn{8}{|l|}{ Evaluation } \\
\hline & Anthesis date & 2004-2005 & 194 & 198 & \multirow[t]{3}{*}{4.36} & \multirow[t]{3}{*}{2.34} & \multirow[t]{3}{*}{-4.33} \\
\hline & \multirow[t]{2}{*}{ (Days after planting) } & $2004-2005$ & 175 & 179 & & & \\
\hline & & 2010-2011 & 189 & 194 & & & \\
\hline & \multirow{3}{*}{$\begin{array}{l}\text { Maturity date } \\
\text { (Days after planting) }\end{array}$} & 2004-2005 & 226 & 231 & \multirow[t]{3}{*}{4.70} & \multirow[t]{3}{*}{2.16} & -4.67 \\
\hline & & 2004-2005 & 206 & 210 & & & \\
\hline & & $2010-2011$ & 219 & 224 & & & \\
\hline & LAl & $2004-2005$ & 5.9 & 5.5 & 0.66 & 12.10 & -0.37 \\
\hline & & 2004-2005 & 5.4 & 6.2 & & & \\
\hline & & 2010-2011 & 5.0 & 5.7 & & & \\
\hline & Biomass $\left(\mathrm{kg} \mathrm{ha}^{-1}\right)$ & $2004-2005$ & 15840 & 14064 & 1240.0 & 19.23 & 564 \\
\hline & & $2004-2005$ & 15424 & 14613 & & & \\
\hline & & 2010-2011 & 14070 & 14965 & & & \\
\hline & Yield $\left(\mathrm{kg} \mathrm{ha}^{-1}\right)$ & $2004-2005$ & 5993 & 5372 & 646.7 & 11.17 & 188 \\
\hline & & 2004-2005 & 6160 & 5530 & & & \\
\hline & & 2010-2011 & 5214 & 5901 & & & \\
\hline
\end{tabular}


Table 5 The average annual temperature and precipitation for the baseline and future climate projection scenarios.

\begin{tabular}{|c|c|c|c|c|c|}
\hline Study regions & Historical and near future & Mean temperature $\left({ }^{\circ} \mathrm{C}\right)$ & *Standard deviation $\left({ }^{\circ} \mathrm{C}\right)$ & Precipitation $(\mathrm{mm})$ & *Standard deviation $(\mathrm{mm})$ \\
\hline \multirow[t]{6}{*}{ Mashhad } & Baseline & 15.62 & - & 241.20 & \\
\hline & $(1990-2019)$ & & & & \\
\hline & RCP 4.5 & 15.75 & 0.49 & 238.57 & 43.73 \\
\hline & $(2021-2050)$ & & & & \\
\hline & RCP 8.5 & 15.93 & 0.58 & 215.04 & 25.68 \\
\hline & $(2021-2050)$ & & & & \\
\hline \multirow[t]{6}{*}{ Neyshabour } & Baseline & 14.22 & - & 238.32 & \\
\hline & (1992-2019) & & & & \\
\hline & RCP 4.5 & 14.50 & 0.46 & 226.46 & 40.91 \\
\hline & $(2021-2050)$ & & & & \\
\hline & RCP 8.5 & 14.67 & 0.57 & 209.18 & 27.85 \\
\hline & $(2021-2050)$ & & & & \\
\hline \multirow[t]{6}{*}{ Sabsevar } & Baseline & 18.09 & - & 184.057 & \\
\hline & $(1990-2019)$ & & & & \\
\hline & RCP 4.5 & 18.30 & 0.44 & 167.56 & 41.99 \\
\hline & $(2021-2050)$ & & & & \\
\hline & RCP 8.5 & 18.51 & 0.57 & 160.39 & 50.37 \\
\hline & $(2021-2050)$ & & & & \\
\hline \multirow[t]{6}{*}{ Torbat-e jam } & Baseline & 17.02 & - & 165.70 & \\
\hline & $(1993-2019)$ & & & & \\
\hline & RCP 4.5 & 18.09 & 0.61 & 162.30 & 53.21 \\
\hline & $(2021-2050)$ & & & & \\
\hline & RCP 8.5 & 18.27 & 0.60 & 148.36 & 58.25 \\
\hline & $(2021-2050)$ & & & & \\
\hline
\end{tabular}

*The standard deviation indicates the variability of projections across the climatic models.

Table 6 Mean simulated days to flowering (anthesis) and maturity over the baseline period (1990-2019) and projected future climates (2021-2051) in the study areas.

\begin{tabular}{|c|c|c|c|c|c|c|}
\hline & Baseline & & RCP4.5 & & RCP8.5 & \\
\hline \multirow[t]{2}{*}{ Location } & $\begin{array}{l}\text { Anthesis } \\
\text { date }\end{array}$ & $\begin{array}{l}\text { Physiological maturity date } \\
\text { (days) }\end{array}$ & $\begin{array}{l}\text { Anthesis } \\
\text { date }\end{array}$ & $\begin{array}{l}\text { Physiological maturity date } \\
\text { (days) }\end{array}$ & $\begin{array}{l}\text { Anthesis } \\
\text { date }\end{array}$ & $\begin{array}{l}\text { Physiological maturity date } \\
\text { (days) }\end{array}$ \\
\hline & (days) & & (days) & & (days) & \\
\hline \multirow[t]{3}{*}{ Mashhad } & 200 & 229 & 203 & 230 & 202 & 228 \\
\hline & & & $(0.9)$ & $(1.1)$ & $(1.7)$ & (2.1) \\
\hline & & & & $(1.1)$ & & \\
\hline \multirow[t]{2}{*}{ Neyshabour } & 204 & 235 & 216 & 244 & 215 & 242 \\
\hline & & & $(1.0)$ & (1.3) & $(2.2)$ & (3.1) \\
\hline \multirow[t]{2}{*}{ Sabzevar } & 190 & 218 & 193 & 219 & 191 & 217 \\
\hline & & & $(1.0)$ & $(1.4)$ & $(2.1)$ & (2.3) \\
\hline \multirow{2}{*}{$\begin{array}{l}\text { Torbat-e } \\
\text { Jam }\end{array}$} & 196 & 225 & 194 & 221 & 193 & 219 \\
\hline & & & $(1.9)$ & $(2.7)$ & $(2.3)$ & (2.5) \\
\hline
\end{tabular}

The values in parentheses are standard error, which indicates the variability of phenological event dates across the climate projections. 
Table 7 Mean growth variables of the irrigated winter wheat under two climate change scenarios (2021-2050) and baseline over different soil textures.

\begin{tabular}{|c|c|c|c|c|c|c|c|c|c|c|c|c|c|}
\hline \multirow[b]{2}{*}{ Location } & \multirow[b]{2}{*}{$\begin{array}{l}\text { Soil } \\
\text { type }\end{array}$} & \multicolumn{4}{|c|}{ Baseline } & \multicolumn{4}{|c|}{ RCP4.5 } & \multicolumn{4}{|l|}{ RCP8.5 } \\
\hline & & $\begin{array}{l}\text { Yield } \\
(\mathrm{kg} \\
\left.\mathrm{ha}^{-1}\right)\end{array}$ & $\begin{array}{l}\text { Fraction of } \\
\text { light } \\
\text { interception } \\
(\%)\end{array}$ & $\begin{array}{l}\text { ET } \\
\text { during } \\
\text { growth } \\
\text { season } \\
(\mathrm{mm})\end{array}$ & LAl & $\begin{array}{l}\text { Yield } \\
\text { (kg ha- } \\
\left.{ }^{1}\right)\end{array}$ & $\begin{array}{l}\text { Fraction of } \\
\text { light } \\
\text { interception } \\
(\%)\end{array}$ & $\begin{array}{l}\text { ET } \\
\text { during } \\
\text { growth } \\
\text { season } \\
(\mathrm{mm})\end{array}$ & LAI & $\begin{array}{l}\text { Yield } \\
(\mathrm{kg} \mathrm{ha}- \\
\left.{ }^{1}\right)\end{array}$ & $\begin{array}{l}\text { Fraction of } \\
\text { light } \\
\text { interception } \\
(\%)\end{array}$ & $\begin{array}{l}\text { ET } \\
\text { during } \\
\text { growth } \\
\text { season } \\
(\mathrm{mm})\end{array}$ & LAl \\
\hline \multirow[t]{8}{*}{ Mashhad } & \multirow{2}{*}{$\begin{array}{l}\text { Clay } \\
\text { Loam }\end{array}$} & \multirow[t]{2}{*}{6105} & \multirow[t]{2}{*}{52.7} & \multirow[t]{2}{*}{527.5} & \multirow[t]{2}{*}{1.84} & 5956 & 52.3 & 514.2 & 1.78 & 5726 & 52.9 & 506.5 & 1.80 \\
\hline & & & & & & *(280) & (3.6) & (1.3) & $(0.16)$ & (202) & (3.7) & (9.2) & $(0.19)$ \\
\hline & \multirow[t]{2}{*}{ Loam } & \multirow[t]{2}{*}{5939} & \multirow[t]{2}{*}{52.1} & \multirow[t]{2}{*}{526.5} & \multirow[t]{2}{*}{1.75} & 5827 & 50.9 & 513.8 & 1.74 & 5672 & 51.2 & 505.2 & 1.74 \\
\hline & & & & & & $(290)$ & (3.8) & (1.4) & $(0.18)$ & (227) & (3.8) & (10.4) & $(0.20)$ \\
\hline & \multirow{2}{*}{$\begin{array}{l}\text { Sandy } \\
\text { clay } \\
\text { loam }\end{array}$} & \multirow[t]{2}{*}{5369} & \multirow[t]{2}{*}{47.2} & \multirow[t]{2}{*}{530.9} & \multirow[t]{2}{*}{1.49} & 5273 & 45.4 & 517.6 & 1.43 & 4917 & 46.9 & 509.3 & 1.50 \\
\hline & & & & & & (304) & (4.1) & (3.6) & $(0.19)$ & (313) & (3.9) & (11.9) & $(0.21)$ \\
\hline & \multirow[t]{2}{*}{ Mean } & 5804 & 50.7 & 528.3 & 1.69 & 5685 & 49.5 & 515.2 & 1.65 & 5438.0 & 50.3 & 507.0 & 1.68 \\
\hline & & (386) & (3.0) & (2.3) & $(0.18)$ & $+(363)$ & (3.6) & (2.1) & (0.19) & (452) & (3.1) & (2.1) & $(0.16$ \\
\hline \multirow[t]{8}{*}{ Neyshabour } & \multirow{2}{*}{$\begin{array}{l}\text { Clay } \\
\text { Loam }\end{array}$} & \multirow[t]{2}{*}{5861} & 47.8 & 557.0 & 1.52 & 6071 & 51.1 & 568.4 & 1.65 & 6226.9 & 51.9 & 557.9 & 1.74 \\
\hline & & & & & & $(241)$ & $(4.4)$ & (7.4) & $(0.28)$ & $(251)$ & (3.7) & $(12.9)$ & $(0.08)$ \\
\hline & Loam & 5677 & 46.7 & 556.1 & 1.45 & 5803 & 49.6 & 567.3 & 1.59 & 6017 & 51.3 & 556.2 & 1.67 \\
\hline & & & & & & $(274)$ & (4.4) & (8.6) & $(0.27)$ & (268) & (3.8) & (13.7) & $(0.07)$ \\
\hline & Sandy & 5039 & 42.6 & 560.8 & 1.21 & 5311 & 44.7 & 569.8 & 1.23 & 5381 & 44.1 & 563.1 & 1.26 \\
\hline & loam & & & & & $(314)$ & $(4.5)$ & (9.4) & $(0.30)$ & (282) & (4.3) & (14.4) & $(0.11)$ \\
\hline & Mean & 5526 & 45.7 & 557.9 & 1.39 & 5728 & 48.5 & 568.5 & 1.49 & 5875 & 49.1 & 559.1 & 1.56 \\
\hline & & (431) & (2.7) & (2.5) & $(0.16)$ & $(385)$ & (3.3) & (1.2) & $(0.23)$ & $(440)$ & (4.3) & (3.6) & $(0.26)$ \\
\hline Sabzevar & Clay & 6466 & 58.7 & 540.1 & 2.44 & 6252 & 58.7 & 524.6 & 2.30 & 5912 & 58.2 & 523.3 & 2.19 \\
\hline & & & & & & (185) & (3.3) & (12.5) & $(0.15)$ & (151) & (3.7) & (15.3) & $(0.16)$ \\
\hline & Loam & 6296 & 56.8 & 539.3 & 2.37 & 6105 & 57.3 & 524.1 & 2.24 & 5855 & 56.2 & 521.4 & 2.09 \\
\hline & & & & & & $(201)$ & (3.4) & $(13.4)$ & $(0.18)$ & (172) & (3.8) & (17.5) & $(0.18)$ \\
\hline & Sandy & 5549 & 51.5 & 543.9 & 1.99 & 5275 & 50.7 & 526.2 & 1.84 & 5089 & 48.1 & 525.9 & 1.73 \\
\hline & loam & & & & & (382) & (4.1) & $(20.4)$ & $(0.21)$ & (275) & $(4.0)$ & $(25.2)$ & $(0.23)$ \\
\hline & Mean & 6104 & 55.7 & 541.1 & 2.27 & 5877 & 55.6 & 524.9 & 2.13 & 5619 & 54.2 & 523.5 & 2.01 \\
\hline & & (488) & (3.7) & (2.5) & $(0.24)$ & (527) & (4.3) & (1. 1) & $(0.25)$ & $(460)$ & (5.3) & (2.3) & $(0.24)$ \\
\hline Torbat-e & Clay & 6365 & 55.7 & 574.0 & 2.05 & 6360 & 60.9 & 537.2 & 2.42 & 6144 & 59.2 & 532.8 & 2.25 \\
\hline & & & & & & (213) & (3.0) & (16.8) & $(0.11)$ & (233) & (3.2) & (18.3) & $(0.18)$ \\
\hline & Loam & 6290 & 54.1 & 572.9 & 1.96 & 6194 & 57.3 & 535.2 & 2.29 & 6022 & 56.2 & 532.2 & 2.12 \\
\hline & & & & & & (228) & (3.1) & (15.0) & $(0.12)$ & (253) & (3.3) & $(19.2)$ & $(0.19)$ \\
\hline & $\begin{array}{l}\text { Sandy } \\
\text { clay }\end{array}$ & 5375 & 48.3 & 579.1 & 1.52 & 5339 & 49.7 & 542.3 & 1.85 & 5029 & 50.5 & 537.1 & 1.85 \\
\hline & loam & & & & & $(380)$ & (3.3) & (19.3) & $(0.16)$ & $(300)$ & (3.9) & $(24.3)$ & $(0.22)$ \\
\hline & Mean & 6010 & 52.7 & 575.3 & 1.84 & 5964 & 55.9 & 538.2 & 2.19 & 5732 & 55.3 & 534.0 & 2.07 \\
\hline & & (551) & (3.9) & (3.3) & $(0.28)$ & (548) & (5.7) & (3.7) & $(0.30)$ & (612) & (4.4) & $(2.7)$ & $(0.20)$ \\
\hline
\end{tabular}

* For each soil type, the values in parentheses are standard error which indicates the variability of the simulated traits across the climate projections.

† For the mean of soil types, the values in parentheses are standard error which indicates the variability of the simulated traits across the soil types. 
Table 8 The response of growth variables in irrigated winter wheat to RCP4.5 and RCP8.5 scenarios of climate change along (2021-2050) with reduced irrigation under different ranges of irrigation efficiency and soil types.

\begin{tabular}{|c|c|c|c|c|c|c|c|c|c|c|c|c|c|}
\hline \multirow{3}{*}{ Location } & & \multicolumn{4}{|c|}{ Irrigation efficiency $\mathbf{4 0} \%$} & \multicolumn{4}{|c|}{ Irrigation efficiency 55\% } & \multicolumn{4}{|c|}{ Irrigation efficiency $70 \%$} \\
\hline & & & \multicolumn{2}{|c|}{$\begin{array}{l}\text { Fraction of light } \\
\text { interception (\%) }\end{array}$} & \multicolumn{2}{|l|}{ LAI } & \multicolumn{2}{|c|}{$\begin{array}{l}\text { Fraction of light } \\
\text { interception (\%) }\end{array}$} & \multicolumn{2}{|l|}{ LAl } & \multicolumn{2}{|c|}{$\begin{array}{l}\text { Fraction of light } \\
\text { interception (\%) }\end{array}$} \\
\hline & & RCP 4.5 & RCP8.5 & RCP4.5 & RCP8.5 & RCP4.5 & RCP8.5 & RCP4.5 & RCP8.5 & RCP4.5 & RCP8.5 & RCP4.5 & RCP8.5 \\
\hline \multirow[t]{8}{*}{ Mashhad } & \multirow{2}{*}{$\begin{array}{l}\text { Clay } \\
\text { Loam }\end{array}$} & 1.77 & 1.70 & 52.0 & 51.2 & 1.81 & 1.79 & 52.7 & 52.4 & 1.82 & 1.87 & 52.2 & 53.6 \\
\hline & & $\star(0.17)$ & $(0.20)$ & (3.6) & (3.8) & $(0.13)$ & $(0.20)$ & $(3.9)$ & (3.8) & $(0.10)$ & $(0.15)$ & (3.6) & $(2.9)$ \\
\hline & \multirow[t]{2}{*}{ Loam } & 1.70 & 1.58 & 50.1 & 48.5 & 1.76 & 1.70 & 51.4 & 50.9 & 1.78 & 1.80 & 51.5 & 50.3 \\
\hline & & $(0.19)$ & $(0.20)$ & $(3.8)$ & (3.8) & $(0.15)$ & $(0.21)$ & (3.8) & (3.8) & $(0.13)$ & $(0.16)$ & (3.8) & (3.3) \\
\hline & \multirow{2}{*}{$\begin{array}{l}\text { Sandy } \\
\text { clay } \\
\text { loam }\end{array}$} & 1.40 & 1.42 & 45.1 & 44.9 & 1.47 & 1.41 & 46.5 & 45.8 & 1.43 & 1.52 & 44.9 & 46.8 \\
\hline & & $(0.22)$ & $(0.23)$ & $(4.4)$ & $(4.4)$ & $(0.17)$ & $(0.23)$ & $(3.5)$ & $(4.3)$ & $(0.15)$ & $(0.18)$ & $(4.0)$ & (3.7) \\
\hline & \multirow[t]{2}{*}{ Mean } & 1.63 & 1.53 & 49.1 & 48.2 & 1.68 & 1.63 & 50.2 & 49.7 & 1.67 & 1.73 & 49.5 & 50.2 \\
\hline & & $+(0.20)$ & $(0.19)$ & (3.6) & $(3.2)$ & $(0.18)$ & $(0.20)$ & (3.3) & (3.5) & $(0.21)$ & $(0.18)$ & $(4.0)$ & (3.4) \\
\hline \multirow[t]{8}{*}{ Neyshabour } & \multirow{2}{*}{$\begin{array}{l}\text { Clay } \\
\text { Loam }\end{array}$} & 1.63 & 1.46 & 50.7 & 48.1 & 1.69 & 1.63 & 52.8 & 51.9 & 1.78 & 1.80 & 52.7 & 53.1 \\
\hline & & $(0.10)$ & $(0.20)$ & $(3.5)$ & $(4.7)$ & $(0.13)$ & $(0.29)$ & (3.6) & (4.5) & $(0.11)$ & $(0.18)$ & (3.5) & $(4.0)$ \\
\hline & \multirow[t]{2}{*}{ Loam } & 1.54 & 1.40 & 49.5 & 46.9 & 1.66 & 1.57 & 52.1 & 50.4 & 1.71 & 1.76 & 52.2 & 51.4 \\
\hline & & $(0.12)$ & $(0.21)$ & $(3.6)$ & $(5.0)$ & $(0.13)$ & $(0.31)$ & (3.7) & $(4.5)$ & $(0.12)$ & $(0.22)$ & (3.7) & $(4.2)$ \\
\hline & \multirow{2}{*}{$\begin{array}{l}\text { Sandy } \\
\text { clay } \\
\text { loam }\end{array}$} & 1.19 & 1.19 & 43.2 & 42.0 & 1.31 & 1.23 & 45.8 & 45.1 & 1.28 & 1.31 & 44.5 & 45.6 \\
\hline & & $(0.15)$ & $(0.29)$ & $(4.6)$ & $(4.5)$ & $(0.15)$ & $(0.32)$ & $(4.5)$ & $(4.5)$ & $(0.14)$ & $(0.23)$ & $(4.2)$ & (3.9) \\
\hline & \multirow[t]{2}{*}{ Mean } & 1.45 & 1.32 & 47.8 & 45.6 & 1.55 & 1.48 & 50.2 & 49.1 & 1.59 & 1.62 & 49.8 & 50.0 \\
\hline & & $(0.23)$ & $(0.19)$ & $(4.0)$ & (3.2) & $(0.21)$ & $(0.22)$ & (3.8) & (3.6) & $(0.27)$ & $(0.27)$ & $(4.6)$ & (3.9) \\
\hline \multirow[t]{8}{*}{ Sabzevar } & \multirow{2}{*}{$\begin{array}{l}\text { Clay } \\
\text { Loam }\end{array}$} & 2.10 & 1.94 & 58.0 & 55.5 & 2.48 & 2.28 & 59.8 & 58.6 & 2.60 & 2.57 & 60.4 & 60.6 \\
\hline & & $(0.15)$ & $(0.24)$ & (3.1) & (3.4) & $(0.16)$ & $(0.16)$ & (3.3) & (3.8) & $(0.14)$ & $(0.17)$ & (3.2) & (3.7) \\
\hline & \multirow[t]{2}{*}{ Loam } & 2.01 & 1.84 & 56.5 & 52.7 & 2.45 & 2.22 & 59.0 & 56.7 & 2.55 & 2.54 & 59.5 & 58.7 \\
\hline & & $(0.17)$ & $(0.14)$ & (3.2) & (3.4) & $(0.18)$ & $(0.18)$ & (3.3) & $(3.9)$ & $(0.16)$ & $(0.19)$ & (3.3) & (3.9) \\
\hline & \multirow{2}{*}{$\begin{array}{l}\text { Sandy } \\
\text { clay } \\
\text { loam }\end{array}$} & 1.70 & 1.68 & 49.5 & 47.1 & 1.93 & 1.81 & 51.5 & 47.9 & 1.86 & 1.84 & 51.1 & 49.4 \\
\hline & & $(0.21)$ & $(0.26)$ & (3.7) & (3.6) & $(0.21)$ & $(0.26)$ & (3.5) & $(5.6)$ & $(0.18)$ & $(0.12)$ & (3.4) & (3.5) \\
\hline & \multirow[t]{2}{*}{ Mean } & 1.94 & 1.82 & 54.6 & 51.8 & 2.29 & 2.10 & 56.8 & 54.4 & 2.32 & 2.33 & 57.0 & 56.2 \\
\hline & & $(0.21)$ & $(0.13)$ & $(4.5)$ & (4.3) & $(0.31)$ & & (4.6) & $(5.7)$ & $(0.40)$ & $(0.41)$ & (5.1) & (5.9) \\
\hline Torbat-e & Clay & 2.16 & 2.10 & 59.0 & 56.8 & 2.50 & 2.41 & 60.7 & 59.6 & 2.69 & 2.54 & 63.1 & 60.1 \\
\hline & & $(0.13)$ & $(0.28)$ & $(2.8)$ & (3.7) & $(0.16)$ & $(0.17)$ & (3.2) & (3.2) & $(0.12)$ & $(0.23)$ & $(2.8)$ & $(2.1)$ \\
\hline & Loam & 2.04 & 1.98 & 56.6 & 54.2 & 2.42 & 2.32 & 58.5 & 56.7 & 2.67 & 2.43 & 61.0 & 57.7 \\
\hline & & $(0.14)$ & $(0.30)$ & $(2.9)$ & (3.8) & $(0.17)$ & $(0.18)$ & $(3.1)$ & (3.6) & $(0.13)$ & $(0.25)$ & $(2.9)$ & (2.3) \\
\hline & Sandy & 1.71 & 1.70 & 49.0 & 48.2 & 1.85 & 1.83 & 50.6 & 48.6 & 1.78 & 1.89 & 49.6 & 48.6 \\
\hline & loam & $(0.18)$ & $(0.37)$ & $(3.2)$ & $(4.2)$ & $(0.21)$ & $(0.23)$ & $(2.9)$ & $(2.6)$ & $(0.20)$ & $(0.30)$ & (3.1) & (3.4) \\
\hline & Mean & 1.97 & 1.93 & 54.9 & 53.1 & 2.26 & 2.22 & 56.6 & 54.9 & 2.38 & 2.29 & 57.9 & 55.5 \\
\hline & & $(0.23)$ & $(0.20)$ & $(5.2)$ & (4.4) & $(0.35)$ & $(0.32)$ & (5.3) & (5.7) & $(0.51)$ & $(0.35)$ & (7.2) & (6.1) \\
\hline
\end{tabular}

* For each soil type, the values in parentheses are standard error which indicates the variability of the simulated traits across the climate projections.

† For the mean of soil types, the values in parentheses are standard error which indicates the variability of the simulated traits across the soil types. 
Table 9 The response of grain yield in irrigated winter wheat to RCP4.5 and RCP8.5 scenarios of climate change (2021-2050) along with reduced irrigation under different ranges of irrigation efficiency and soil types.

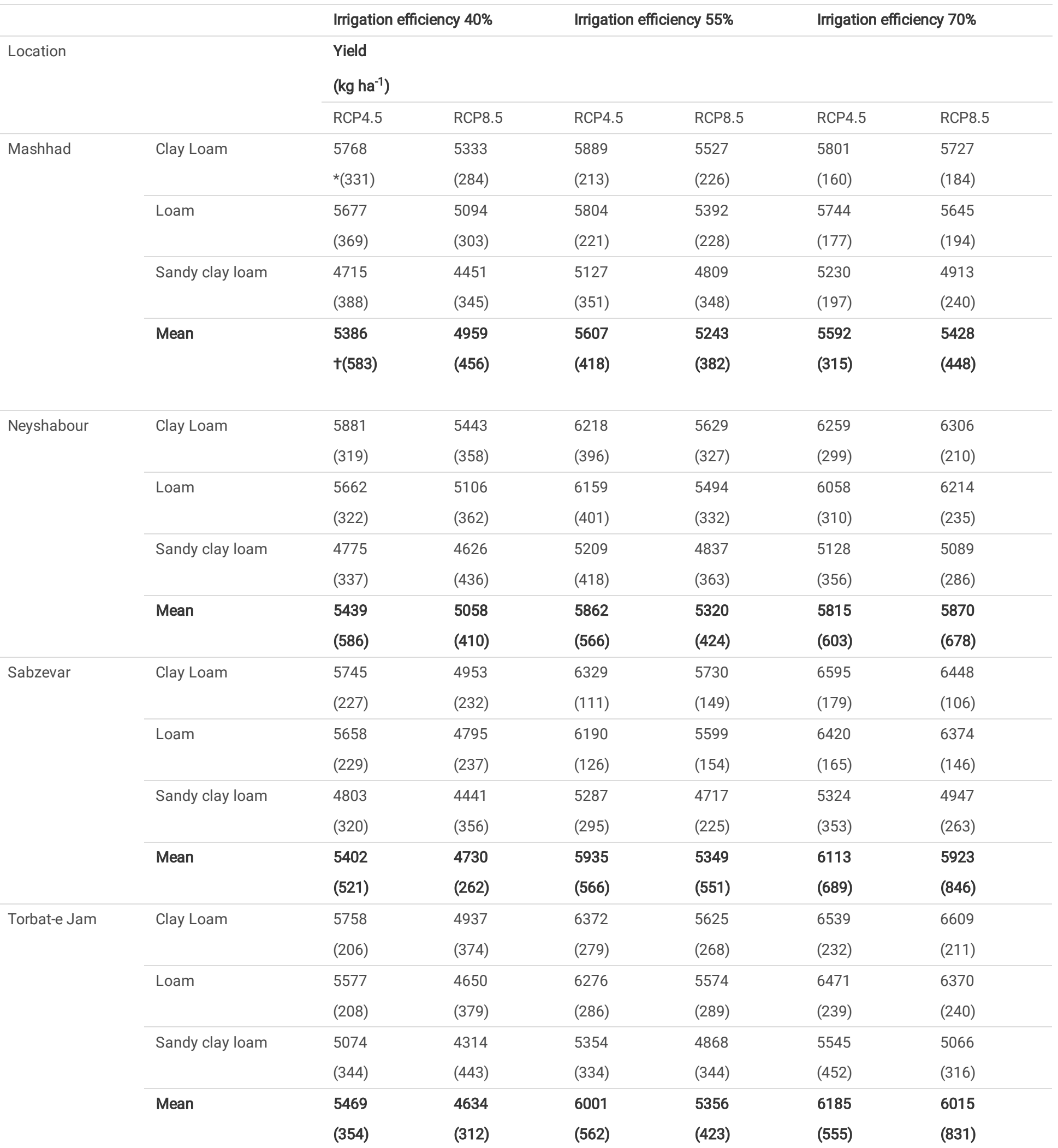

* For each soil type, the values in parentheses are standard error which indicates the variability of the simulated traits across the climate projections.

† For the mean of soil types, the values in parentheses are standard error which indicates the variability of the simulated traits across the soil types.

\section{Results}

Crop model calibration and evaluation 
The CSM-CERES-Wheat model accurately simulated the development, growth, and yield of winter wheat in response to planting dates and irrigation regimes across the study areas (Table 4). The model calibration and validation results showed a good match between the measured and simulated grain yield with the nRMSE values ranged from 5.6 to $11.2 \%$. The standard deviation of the yield prediction errors (RMSE) ranged from $376 \mathrm{~kg} \mathrm{ha}^{-1}$ in calibration to $647 \mathrm{~kg}^{-1}$ in evaluation. The MBE values showed that the yield was slightly underestimated $\left(-85 \mathrm{~kg} \mathrm{ha}^{-1}\right)$ during the calibration phase and overestimated (+188 $\left.\mathrm{kg} \mathrm{ha}{ }^{-1}\right)$ during the evaluation phase. The crop model performance for simulating total aboveground biomass was good during both phases, with RMSE, NRMSE, and MBE values of 778-1240 $\mathrm{kg} \mathrm{ha}^{-1}, 4.7-19.2 \%, 10.3-564 \mathrm{~kg} \mathrm{ha}^{-1}$. The maximum leaf area index was simulated well (Table 4). The RMSE and nRMSE values for leaf area index ( $\mathrm{LAl}$ ) were $0.48-0.66$ and $7.40-12.10 \%$, respectively. The MBE values showed that the model tended to underestimate LAI during the calibration and evaluation phases. The model performance for simulating the anthesis and maturity dates was excellent during both assessment phases, with RMSE and nRMSE values of 2.1-4.7 days and 1.1-2.34 \%, respectively. Furthermore, the anthesis and maturity dates were underestimated about 2.0 days during the calibration phase and 4.5 days during the evaluation phase (Table 4).

\section{Past, present, and future climate characteristics}

Since the 1900s, the average annual temperature changes can be divided into three time periods for Iran (Fig 2.). The temperature changes showed no trend from 1901 to1930, while two warming periods have experienced with different increasing rates since the 1930s. The annual temperature increased by an average rate of $0.08^{\circ} \mathrm{C}$ per decade from 1930 to 1986 and $0.45^{\circ} \mathrm{C}$ per decade from 1986 to 2016. The annual precipitation showed an increasing trend of 1.9 mm per decade from 1901 to 1987 and has followed by a decreasing rate of about $18 \mathrm{~mm}$ per decade since 1988 (Fig. 2).

Analysis of weather data over the past 27-30 years (Fig. 3), as the baseline period, indicated that the average annual temperatures increased from 0.34 to $0.79{ }^{\circ} \mathrm{C}$ per decade across the study regions. The rising rate of temperature was the highest in Mashhad, followed by Torbat-e Jam, Neyshabour, and Sabzevar. The annual precipitations in the baseline period have shown an overall decreasing trend in most study areas, ranging from 13.2 to $16.3 \mathrm{~mm}$ per decade (Fig.

3). However, the annual precipitation in Neyshabour showed an increasing trend with a rate of $1.54 \mathrm{~mm}$ per decade. For the near-future (2021-2050) under the RCP4.5 scenario, average temperatures are projected to increase by $0.3,0.28,0.21$, and $1.07{ }^{\circ} \mathrm{C}$ relative to the baseline period in Mashhad, Neyshabur, Sabsevar and Torbat-e jam, respectively (Table 5). Under the RCP8.5 scenario, projections suggest that air temperatures will increase by $0.31,0.45,0.42$, and $1.25^{\circ} \mathrm{C}$ in Mashhad, Neyshabour, Sabsevar, and Torbat-e jam, respectively. In Mashhad, Neyshabur, Sabsevar, and Torbat-e Jam, the annual precipitations are projected to decrease, respectively, by $2.63,11.86,16.50$, and $3.40 \mathrm{~mm}$ under the RCP4.5 scenario; and also by $26.16,29.14,23.67$, and $17.34 \mathrm{~mm}$ under the RCP8.5 scenario in the near future (Table 5).

Future climate projections also indicate that the mean temperature during the wheat vegetative growth period (about the first 180 days after planting) will decrease by $1.53-1.69^{\circ} \mathrm{C}$ under RCP.4.5 and $1.24-1.37^{\circ} \mathrm{C}$ under RCP.8.5 across the study areas, except Torbat-e Jam (Fig. 4). In Torbat-e Jam, the mean temperature during the vegetative growth period will increase by 1.15 and $1.36^{\circ} \mathrm{C}$ under RCP 4.5 and RCP8, respectively. The mean temperature under different climate scenarios will increase $1.74-2.73^{\circ} \mathrm{C}$ during the whole reproductive growth period (about 180 to 226 days after planting) in most study areas, except in Neyshabour (Fig. 4). In Neyshabour, the rising temperature will coincide with the end of the grain-filling period (beyond 210 days after planting). Analysis of precipitation projections indicates that the precipitation rate in most of the growing season (the first 210 days after planting) will decrease by $22.6-51.9$ $\mathrm{mm}$ under RCP4.5 and 17.5-60.8 mm under RCP8.5 across the study areas (Fig. 5). However, the precipitation rates will increase during the end of the grainfilling period in all regions.

Climate change effects under the current irrigation water availability

Climate impact on wheat phenology

The length of the period from sowing to flowering, known as the vegetative growth period, and days from flowering to maturity, known as the grain-filling period, are two crucial events in the plant life cycle. The simulation results showed that soil texture types did not affect flowering and maturity dates, so the simulated days to phenological events were averaged across soil types and shown in Table 6. For the baseline period (1990-2019), the mean simulated days to flowering and maturity ranged from 190 and 218 in Sabzevar to 204 and 235 in Neyshabour, respectively. The historical changes of winter wheat phenology showed that mean simulated length of the vegetative growth and grain filling periods have decreased linearly in the study areas (Fig. 6). For example, the mean flowering dates advanced by 4.2, 2.5, 1.9, and 4.4 days per decade in Mashhad, Neyshabour, Sabzevar, and Torbat-e Jam, respectively (Fig. 6). Furthermore, the days to maturity decreased by $4.3,2.7,2.1$, and 4.5 days per decade in Mashhad, Neyshabour, Sabzevar, and Torbat-e Jam, respectively. The mean simulated grain filling duration also has decreased over the baseline period, with the lowest reduction trend in Neyshabour and the highest reduction in Torbat-e Jam (Fig. 6).

Results also indicated that the impact of near-future climate change on wheat phenology would vary depending on the locations (Table 6). The mean length of the vegetative growth period over the near future will increase by 3, 12, and 3 days under RCP4.5, and 2, 11, and 1 days under RCP8.5 in Mashhad, Neyshabour, and Sabzevar, respectively. In Torbat-e Jam, the simulated length of the vegetative growth period will decrease by 2 and 3 days under RCP4.5 and RCP8.5, respectively (Table 6). However, the whole growth period will be shorted by 1-6 days under the RCP8.5 climate scenario relative to the baseline in Mashhad, Sabzevar, and Torbat-e Jam. For both of the RCP scenarios, reduction in grain filling duration ranged from 2 to 4 days for the near-future climate relative to baseline across the study areas. Furthermore, the inter-models variability of the simulated dates to flowering and maturity ranged from 0.9 to 3.1 days under both RCPs.

Climate impacts on LAl and light interception

The simulated LAI and light interception varied by locations, climate models, and RCP scenarios. In the baseline period, the highest and lowest values for the mean simulated LAl and fraction of light interception during the growing season corresponded to Sabzevar and Neyshabour, respectively (Table 7). For the 
future scenarios, the simulated seasonal LAl varied between 1.23 and 2.30 in the study areas, with a mean standard deviation of 0.23 across the different soil types. The mean seasonal LAl decreased by 2.6 and $6.2 \%$ under RCP4.5 and 0.8 and $11.6 \%$ under RCP8.5 compared with the baseline in Mashhad and Sabzevar, respectively. The mean seasonal LAl increased by 6.9 and $19.0 \%$ under RCP4.5 and 11.7 and $12.5 \%$ under RCP8.5 compared with the baseline in Neyshabour and Torbat-e jam, respectively. LAl variability across the different soil textures ranged from 0.16 to 0.28 in the baseline period and from 0.16 to 0.30 in the future scenarios. Under the future climate relative to the baseline climate, the seasonal fraction of light interception increased on average by 6.7 and $5.6 \%$ in Neyshabour and Torbat-e Jam; and decreased on average by 1.5 and $1.4 \%$ in Mashhad and Sabzevar, respectively.

Climate impact on potential evapotranspiration (PET)

The total PET during the winter wheat-growing season was affected by locations, climate models, and RCP scenarios. The effect of soil texture on the PET variability was negligible, and the variability ranged from 1.1 to $3.7 \mathrm{~mm}$ across the soil textures in the study areas (Table 7). The mean growing season PET ranged about from $528 \mathrm{~mm}$ in Mashhad to $564 \mathrm{~mm}$ in Torbat-e Jam over the baseline period. The trend analysis showed that the growing season evapotranspiration decreased linearly over the bassline period (Fig. 7). The reduction rates were 17.8, 14.0, 6.0, and 17.9 mm per decade in Mashhad, Neyshabour, Sabzevar, and Torbat-e Jam, respectively. Furthermore, the projected PET decreased by 13.1, 16.2, and 37.1 mm under RCP4.5 and 21.3, 17.6, and $41.3 \mathrm{~mm}$ under RCP8.5 relative to the baseline period in Mashhad, Sabzevar, and Torbat-e Jam, respectively (Table 7). The growing season PET increased by 10.6 and $1.2 \mathrm{~mm}$ under RCP4.5 and RCP8.5 relative to the baseline in Neyshabour. The mean inter-climate model variability in the projected PET ranged from 2.1 to $17.0 \mathrm{~mm}$ under RCP4.5 and 10.5 to $20.6 \mathrm{~mm}$ under RCP8.5 across the study areas.

Climate impact on wheat yield productivity

The simulated winter wheat production varied by locations, climate models, RCP scenarios, and soil types. The simulation results showed that the highest yield potential $\left(6104 \mathrm{~kg} \mathrm{ha}^{-1}\right)$ was obtained in Sabzevar, and the lowest yield potential $\left(5526 \mathrm{~kg} \mathrm{ha}^{-1}\right)$ was obtained in Neyshabour over the baseline period (Table 7). The simulated yields in the clay and loam soils were notably greater than in the sandy clay loam soil across all sites. The standard deviation of historical yield across the selected soil types ranged from $386 \mathrm{~kg} \mathrm{ha}^{-1}$ in Mashhad to $551 \mathrm{~kg} \mathrm{ha}^{-1}$ in Torbat-e Jam (Table 7). The simulated yields have increased on average by $272,155,133$, and $143 \mathrm{~kg}^{-1}$ per decade over the baseline period in Mashhad, Neyshabour, Sabzevar, and Torbat-e Jam, respectively (Fig. 7). However, the projected yield will decrease under the near-future climate change scenarios relative to the baseline period in Mashhad, Sabzevar, and Torbat-e jam (Table 7). The average reduction in the projected yields were 2, 3.7, and 0.8 \% for the RCP4.5 and 6.3, 7.9, and 4.6\% for the RCP8.5 in Mashhad, Sabzevar, and Torbat-e Jam, respectively. In Neyshabour, the projected yield increased $3.7 \%$ under the RCP4.5 and $7.9 \%$ under the RCP4.5 compared with the contemporary scenario (Table 7). The mean inter-climate model variability of the projected wheat yield ranged from 256 to $277 \mathrm{~kg}^{-1} \mathrm{under}^{-1} \mathrm{Re}$ RCP4.5 and 199 to $267 \mathrm{~kg} \mathrm{ha}^{-1}$ under the RCP8.5 across the study areas. The standard deviation of the projected yields across the soil types ranged from $363 \mathrm{~kg}^{-1}$ in Mashhad to $612 \mathrm{~kg} \mathrm{ha}^{-1}$ in Torbat-e Jam under future climate projections (Table 7).

Climate change effects under irrigation water shortage and adaptation strategy

Water scarcity is the key challenge in arid regions, which exacerbates under $\mathrm{CC}$, and must be considered to make an accurate projection of climate change impacts on crop production. We quantified the impact of CC on irrigation water availability by using FAO data on the internal renewable water resources, precipitation, and reference evapotranspiration at countries' level (Fig. 8). The nonlinear regression analysis showed that IRWR increased with increasing the ratio of $\mathrm{P}$ to $\mathrm{ET}$ 。 $\left(\mathrm{R}^{2}=0.81\right.$ and $\left.\mathrm{RMSE}=0.74\right)$. Based on the obtained relationship, the future renewable water resources on average were projected to decrease by $14.4 \%$ under RCP 4.5 and $25.4 \%$ under RCP.8.5 across the study areas. Therefore, we re-analyzed the impacts of CC on wheat growth under the projected irrigation water shortages and finally evaluated the role of irrigation efficiency (IE) in minimizing water shortage impacts.

At the current irrigation efficiency (40\%), the mean seasonal LAl decreased by 3.0, 7.0, 3.6, and $4.8 \%$ for RCP4.5 and 7.3, 11.4, 14.6 , and $11.9 \%$ for RCP8.5 under the projected future irrigation shortage compared with the current irrigation levels in Mashhad, Neyshabour, Sabzevar, and Torbat-e Jam, respectively (Table 8). At similar irrigation efficiency, the mean seasonal fraction of light interception decreased by $1.5 \%$ for RCP4.5 and $4.9 \%$ for RCP.8.5 under the future irrigation shortage compared with the current irrigation levels across the study areas. Under the future water scarcity conditions, the mean seasonal LAI will increase by $3.5,6.9,12.8$, and $9.5 \%$ for RCP4.5 and 6.5, 11.9, 15.6, and $13.3 \%$ for RCP8.5 with improving IE from 40 to $55 \%$ in Mashhad, Neyshabour, Sabzevar and Torbat-e Jam, respectively. At similar conditions, mean seasonal LAI will increase by 2.4-20.8\% under RCP.4.5 and 13.1-28.0\% under RCP.8.5 with improving IE from 40 to $70 \%$ across the study areas. The mean seasonal fraction of light interception under the limited irrigation conditions increased by $3.6 \%$ for RCP4.5 and $4.8 \%$ for RCP 8.5 with improving IE from 40 to $55 \%$, and also $3.7 \%$ for RCP 4.5 and $6.7 \%$ for RCP 8.5 with improving IE from 40 to $70 \%$ over the study areas (Table 8).

Winter wheat yield potential declined by 5.2, 5.0, 8.1, and 8.3\% for RCP4.5 and 8.8, 13.9, 15.8, and $19.2 \%$ for RCP8.5 under the projected irrigation shortage compared with the current irrigation levels in Mashhad, Neyshabour, Sabzevar, and Torbat-e Jam, respectively (Table 9). Under reduced irrigation conditions, grain yield increased by 4.1-9.9\% for RCP4.5 and 5.2-15.6\% for RCP8.5, with improving IE from 40 to $55 \%$ across the study areas. The grain yield also increased by 3.8-13.2\% for RCP4.5 and 9.5-29.8\% for RCP8.5, with increasing irrigation efficiency from 40 to $70 \%$ (Table 9 ). The variation in the projected yield due to different soil types ranged from 263 to $846 \mathrm{~kg} \mathrm{ha}^{-1}$ across the study areas.

\section{Discussion}

Any change in climate conditions can affect climate-sensitive systems such as agriculture, pastoralism, forestry, and other natural resources (IPCC, 2014) and undoubtedly challenge food production (El-Beltagy and Madkour 2012). Analysis of historical temperature data in Iran over the period $1901-2016$ showed a plateauing trend from 1901 to 1930 , followed by two warming periods with an average rate of 0.08 and $0.45^{\circ} \mathrm{C}$ per decade for $1930-1986$ and $1986-2016$, 
respectively. Furthermore, the annual precipitation showed an increasing trend with an average rate of $1.9 \mathrm{~mm}$ per decade from 1901 to 1987 and has been followed by a decreasing rate of about $18 \mathrm{~mm}$ per decade since 1988. It was reported that the global annual temperature has increased at an average rate of $0.08^{\circ} \mathrm{C}$ per decade since 1880 and over twice that rate $\left(+0.18^{\circ} \mathrm{C}\right.$ ) since 1981 (NOAA Global Climate Report 2020). During the 1910-2020 period, the warming trend in Asia was $+0.17^{\circ} \mathrm{C}$ per decade; however, the $1981-2020$ trend was $+0.37^{\circ} \mathrm{C}$ per decade (NOAA Global Climate Report 2020). Both increasing and decreasing precipitation trends were observed for different parts of Asia, although a downward trend in mean precipitation was observed for recent decades in west Asia (Hijioka et al. 2014).

For the near-future (2021-2050), the average annual temperatures are projected to increase by $0.3-1.07^{\circ} \mathrm{C}$ under mid-range emissions scenario (RCP4.5) and $0.31-1.25^{\circ} \mathrm{C}$ under high-emissions scenario (RCP8.5), relative to the baseline over the study areas. Furthermore, the annual precipitations are projected to decrease by $2.6-16.5 \mathrm{~mm}$ under RCP4.5 and 17.3-29.1 mm under RCP8.5 relative to the baseline. Near-future climate projections indicated that the mean temperature during the vegetative wheat growth period would decrease by $1.24-1.69^{\circ} \mathrm{C}$ compared with the baseline period in the study areas, except Torbat-e Jam. For Torbat-e Jam, the mean temperature during the vegetative wheat growth period will increase by $1.15-1.36^{\circ} \mathrm{C}$ in the near future. However, the mean temperatures are projected to increase at rates of $1.74-2.73{ }^{\circ} \mathrm{C}$ during the reproductive wheat growth period over the study areas. The precipitation projections also indicated that the precipitation rates would decrease over most of the wheat growing period, and increase during the end of the grain-filling period in all regions. Shojaee and Fallah-Ghalhari (2017) reported that the average annual temperatures increased by $0.75^{\circ} \mathrm{C}$ per decade, and the average annual precipitations decreased by $26.1 \mathrm{~mm}$ per decade from 1991 to 2015 at eight stations across the Khorasan Razavi province.

Our results obtained by simulating wheat production under future climate projections with current irrigation supply showed that yield would decrease by $0.8-$ $7.9 \%$ in three regions and increase $3.7-7.9 \%$ in Neyshabour. It has been projected that irrigated wheat production might decrease by $0.44-33.7 \%$ or increase by $0.19-9.2 \%$ under future climate conditions in different regions of Iran (Valizadeh et al. 2014, Karimi et al. 2018). Increased temperatures during the reproductive stage reduced the grain-filling period (Table 6), and finally, yield production decreased due to the reduced time available for photosynthesis activity. The optimum temperature for wheat anthesis and grain-filling stages ranges from 12 to $22^{\circ} \mathrm{C}$ (Shewry 2009; Farooq et al. 2011), while the average temperature during the reproductive growth period was about $24^{\circ} \mathrm{C}$ over the regions with decreased yield production. It also has been reported that high temperature affected yield by reducing radiation use efficiency through its negative impact on the structure and function of photosynthetic apparatus (Reynolds et al. 2007; Farooq et al. 2011).

Considering potential changes in future irrigation water availability showed that the relative yield losses were about $6.7-14.4 \%$ under future climate scenarios with deficit irrigation conditions compared to the current irrigation conditions. A mean overall reduction in winter wheat yield due to future climate change would be about $12.3 \%$ across the study areas, which the contribution of heat and drought stress to yield reductions was 1.7 and $10.6 \%$, respectively. However, the decreased yield could be up to $19.2 \%$ in the drier region of the study domain. All studied regions showed increased precipitation during the grain-filling period for the future climate than the present climate (Fig. 5), which could downweight the negative effect of deficit irrigation on yield production. It was reported that the irrigated winter wheat yield reduced on average by $3 \%$ with rising temperature under future climate change (2040-2070) in some arid and semi-arid regions of south Iran (Eyni-Nargeseh et al. 2019). In Mexico, wheat production is projected to decrease by $5.4 \%$ under mid-century climate change (Hernandez-Ochoa et al. 2018). Spring wheat yield would decrease by $4.8-7.8 \%$ with rising temperatures in the range of $1-2{ }^{\circ} \mathrm{C}$ under Egypt's climate, and the reduction could increase to $15.3 \%$ when the heat stress was combined with water deficit stress (Ding et al. 2021).

The impact of near-future climate conditions on wheat phenology would vary depending on temperature changes during the growing seasons in each location. The mean length of the vegetative growth period will extend in the three regions, where wheat plants will experience a lower temperature during the vegetative period in the future than in the baseline period. However, the grain-filling duration will reduce by about 2-4 days in the near-future climate relative to the baseline period across the four locations. The length of the phenological phases, the vegetative and reproductive stages, are controlled by day length and temperature (Robertson 1968; Hemming et al. 2008). Due to the small difference (a couple of minutes) in day length among the studied sites, it seems that the importance of temperature on the flowering date was much higher than the day length. The shortened phenophase is linked to faster crop development under higher temperatures due to a faster rate of thermal time accumulation (Hemming et al. 2008). The grain-filling duration could reduce by $2.4 \mathrm{~d}$ for every $1 \circ \mathrm{C}$ above the optimal growing temperature (Yin et al. 2009). Duration of the grain-filling period has been suggested as a determinant factor of productivity in wheat (Evans and Fischer 1999), as a shorter duration of grain-filling can potentially decrease the length of photosynthetic activity and consequently grain weight (Gebbing et al. 1999; Sommer et al. 2003).

LAl is a good indicator of vegetation status representing the active photosynthetic area and the area subjected to transpiration. The simulations under the current irrigation supply showed that mean seasonal LAI and fraction of light interception increased in Neyshabour and Torbat-e Jam and decreased in Mashhad and Sabzevar for the near-future climate relative to the baseline climate. However, the mean seasonal LAI and fraction of light interception, respectively, decreased by 8.0 and $3.2 \%$ under future climate with deficit irrigation conditions compared to the current irrigation conditions. The increased future precipitation and mean temperature during the early vegetative growth stage (0-90 days after planting, Fig. 4 and 5) could explain the increased LAl and fraction of light interception in Neyshabour and Torbat-jam. It was reported that the rate of wheat leaf appearance was increased by raising the temperature in a range of $2-22{ }^{\circ} \mathrm{C}$ (White et al. 2012) and decreased by water stress (Jiang et al. 2020). The CERES-Wheat has no heat stress factor for leaf senescence (Keating et al. 2001) and the model underestimated leaf senescence at the grain-filling period under heat stress (Liu et al. 2016). Therefore, the effect of high temperatures on leaf senescence rate during the grain-filling period was not included in leaf area calculations, and simulated LAI was more accurate in the vegetative growth period than in the reproductive growth period.

Under near-future climate scenarios relative to the baseline, the projected growing season PET decreased by 13.1-41.3 mm in Mashhad, Sabzevar, and Torbat-e Jam and increased by $1.2-10.6 \mathrm{~mm}$ in Neyshabour. The PET represents the estimate of atmospheric evaporative demand and depends on meteorological factors such as solar radiation, temperature, wind speed, and air humidity (Shirmohammadi-Aliakbarkhani and Saberali 2020). However, not only meteorological factors but also the length of crop-growing period affect the accumulated PET throughout the growing season (Shirmohammadi-

Page 13/23 
Aliakbarkhani, and Saberali 2020). The lower temperature during most of the vegetative period in Mashhad and Sabzevar (Fig. 4) and the shortened length of the growing season in Torbat-e Jam (Table 5) could explain the reductions in PET under near-future climate relative to the baseline climate. Despite the reduced growing season PET, irrigation water requirement (the difference between winter wheat evapotranspiration and precipitation) is expected to increase due to uneven temporal distribution of precipitation and a further reduction in the rate of precipitation than evapotranspiration during most of the growing season under the future climate.

Improved irrigation efficiency has been introduced as the most common worldwide on-farm adaptation strategy to adapt to drought under climate change (Smit and Skinner 2002; Bijl et al. 2018). Under limited irrigation conditions, the mean yield potential increased by $7.8-9.9 \%$ with improving IE from 40 to $55 \%$ and $9.2-20.1 \%$ with improving IE from 40 to $70 \%$ in future climate scenarios. The results showed that the increased yield associated with improving irrigation efficiency was about $9 \%$ greater in drier regions (Torbat-e Jam and Sabzevar) than wetter regions (Mashhad and Neyshabour). An increase of 15 and $30 \%$ in the irrigation efficiency will offset yield reductions associated with limited irrigation supply under the RCP4.5 and RCP8.5 scenarios, respectively. A global analysis of future water deficit (2050s) showed that the overall reductions in water demand with improving water use efficiency are large enough almost to eliminate water deficits and reduce water withdrawal. (Bijl et al. 2018). Water supply is the major limitation to agriculture in arid and semi-arid regions; thus, CC adaptation policies should include developing and deploying improving water management practices to conserve water at the farm and regional scales. Around 100,000 hectares of wheat land are irrigated through surface irrigation systems in Khorasan Razavi province. The cost of changing the irrigation method from a surface to a high-efficiency system (sprinkler and drip irrigation systems) is approximately $\$ 1,000$ per hectare in Iran. Therefore, an investment of $\$ 100$ million in the province and about \$one billion in the whole country over the next years is needed to improve the efficiency of irrigation systems in wheat lands.

Improved irrigation efficiency can provide an opportunity for adaptation to CC, but at a cost and with possible negative side effects (impacts on soil quality). In practical application, high-efficiency irrigation systems can only serve as an adaptation in large-scale wheat fields since the income of smallholder farmers is not sufficient to cover the investment costs of such systems. Furthermore, soil salinity will likely increase in response to warming and drought in arid and semi-arid areas due to increased evapotranspiration and decreased leaching flow rate (Corwin 2021). The salinization process can also be affected by the quantity and quality of irrigation water applied and the irrigation methods used in irrigated lands. As a result of climate change, soil and irrigation water salinity is expected to increase in the arid and semi-arid regions of Iran (Akbari et al. 2020). Irrigating with saline water adds salt to the soil and increases the irrigation water need to leach salts; thus, reduced water consumption in high-efficiency irrigation systems has the potential to increase soil salinity (Malash et al. 2008; Corwin 2021). Therefore, increased irrigation efficiency can only serve as a temporary adaptation strategy in large-scale farms with enough financial support and will not have the practical capacity to adequately adapt to long-term CC due to the increasing soil and groundwater salinity in arid regions.

\section{Conclusion}

The CC has challenged food security by affecting crop production and become a critical global and national issue. Predicting the potential production of crops under CC is critical for finding adaptation strategies to secure sustainable food security under changing climate. Our study used a climate-crop modelling approach to quantify the impact of climate change on irrigated wheat production in some arid regions of northeast Iran. The results demonstrate that Iran's climate has become hotter and drier in recent decades, and this pattern will continue in the future. Future projections indicated that the precipitation rates would decrease over most of the winter wheat growing period, and temperature would increase during the reproductive growth period across the study areas. Furthermore, the future renewable water resources were projected to decrease on average by 14.4 and $25.4 \%$ under the RCP4.5 and RCP.8.5 scenarios, respectively.

The simulation results showed that the mean reduction in winter wheat yield due to future climate change would be about $12.3 \%$ across the study areas, which the contribution of heat and drought stress to yield reductions were 1.7 and $10.6 \%$, respectively. However, the decreased yield could be up to $19.2 \%$ in the drier region of the study domain. The decreased seasonal LAl, the fraction of light interception, and the duration of the grain-filling period under future climate conditions could potentially lead to a decrease in yield production. The seasonal PET is expected to decrease, but irrigation water requirement is expected to increase due to a further reduction in the rate of precipitation than evapotranspiration during the winter wheat growing season. Furthermore, an increase of $15-30 \%$ in irrigation efficiency was sufficient to eliminate the vulnerability of wheat production to drought under CC in arid regions. It is important to note that this study may over-estimate the potential gains of improved irrigation efficiency, because the analyzes did not incorporate the negative impact of CC on soil and groundwater salinity. However, the findings can offer a scientific basis to convince policy makers and farmers that using a high-efficiency irrigation system is a priority to mitigate the adverse effects of future climate change in arid regions.

\section{Declarations}

Acknowledgments This work has been financially supported by the vice-chancellor for research of the University of Torbat-e Jam.

Author contribution S. F. Saberali: Conceptualization, methodology, model validation and evaluation, analyzed and interpreted the climate and crop data, writing-original draft; Zahra Shirmohammadi-Aliakbarkhani and Hossein Nastari Nasrabadi: Data preparation and curation, mapping and visualization, writing-review, and editing.

Funding This study was funded by the University of Torbat-e Jam.

Data availability All the data used for this study are available and provided by the corresponding author on reasonable request.

Code availability DSSAT v4.7. 
Competing interests The authors declare no competing interests.

Ethics approval Not applicable.

Consent to participate Not applicable.

Consent for publication Not applicable.

\section{References}

Agricultural Statistical Yearbook (2018) Ministry of Agriculture Jihad. Agriculture Jahad publication, Iran.

Akbari M, Najafi Alamdarlo H, Mosavi SH (2020) The effects of climate change and groundwater salinity on farmers' income risk. Ecol Indic $110: 105893$. https://doi.org/10.1016/j.ecolind.2019.105893.

Bijl DL, Biemans H, Bogaart PW, Dekker SC, Doelman JC, Stehfest E, van Vuuren DP (2018) A global analysis of future water deficit based on different allocation mechanisms. Water Resour Res 54: 5803-5824. https://doi.org/10.1029/2017WR021688.

Bucchignani E, Mercogliano P, Panitz HJ, Montesarchio M (2018) Climate change projections for the Middle East-North Africa domain with COSMO-CLM at different spatial resolutions. Adv Clim Chang Res. 9: 66-80. https://doi.org/10.1016/j.accre.2018.01.004.

Corwin DL (2021) Climate change impacts on soil salinity in agricultural areas. Eur J Soil Sci 72: 842-862. https://doi.org/10.1111/ejss.13010.

Dalin C, Wada Y, Kastner T, Puma MJ (2017) Groundwater depletion embedded in international food trade. Nature 543: 700-

704. https://doi.org/10.1038/nature21403.

Dettori M, Cesaraccio C, Duce P (2017) Simulation of climate change impacts on production and phenology of durum wheat in Mediterranean environments using CERES-Wheat model. Field Crops Res. 206: 43-53. https://doi.org/10.1016/j.fcr.2017.02.013.

Ding Z, Ali EF, Elmahdy AM, Ragab KE, Seleiman MF, Kheir AMS (2021) Modeling the combined impacts of deficit irrigation, rising temperature and compost application on wheat yield and water productivity. Agric Water Manag 244: 106626. https://doi.org/10.1016/j.agwat.2020.106626.

Doulabian S, Golian S, Toosi AS, Murphy C (2020) Evaluating the effects of climate change on precipitation and temperature for Iran using RCP scenarios. J Water Clim Chang 12: 166-184. https://doi.org/10.2166/wcc.2020.114.

El-Beltagy A, Madkour M (2012) Impact of climate change on arid lands agriculture. Agric. Food Secur. 1: 3-14. https://doi.org/10.1186/2048-7010-1-3.

Eyni-Nargeseh H, Deihimfard R, Rahimi-Moghaddam S, Mokhtassi-Bidgoli A (2020) Analysis of growth functions that can increase irrigated wheat yield under climate change. Meteorol Appl 27: 1-10. https://doi.org/10.1002/met.1804.

FAO/IIASA/ISRIC/ISS-CAS/JRC (2012) Harmonized World Soil Database (Version 1.2). FAO and IIASA, Rome, Italy and Laxenburg, Austria. http://webarchive.iiasa.ac.at/Research/LUC/External-World-soil database/HTML/index. html?sb=1. Accessed 02 December 2021

Farooq M, Bramley H, Palta JA Siddique KHM (2011) Heat stress in wheat during reproductive and grain-filling phases. Crit Rev Plant Sci 30:491507. https://doi.org/10.1080/07352689.2011.615687

Gebbing T, Schnyder H, Kuhbauch W (1999) The utilization of pre-anthesis reserves in grain filling of wheat: assessment by steady-state ${ }^{13} \mathrm{CO} 2 /{ }^{12} \mathrm{CO} 2$ labelling. Plant Cell Environ 22: 851-858. https://doi.org/10.1046/j.1365-3040.1999.00436.x.

Hawkins E, Sutton R (2011) The potential to narrow uncertainty in projections of regional precipitation change. Clim Dyn 37: $407-418$. https://doi:10.1007/s00382-010-0810-6.

Hemming MN, Peacock WJ, Dennis ES, Trevaskis B (2008) Low-temperature and daylength cues are integrated to regulate FLOWERING LOCUS T in barley. Plant Physiol 147: 355-366. https://doi.org/10.1104/pp.108.116418.

Hernandez-Ochoa I.M, Asseng S, Kassie BT, Xiong W, Robertson R, Luz Notelo Pequeno D, Sonder K, Reynolds M, Babar MA, Molero-Milan A, Hoogenboom, G (2018) Climate change impact on Mexico wheat production. Agric. For Meteorol 263: 373-387. https://doi.org/10.1016/j.agrformet.2018.09.008.

Hijioka Y, Lin E, Pereira JJ, Corlett RT, Cui X, Insarov GE, Lasco RD, Lindgren E, Surjan A (2014) Asia, In: Barros VR, Field CB, Dokken DJ, Mastrandrea MD, Mach KJ, Bilir TE, Chatterjee M, Ebi KL, Estrada YO, Genova RC, Girma B, Kissel ES, Levy AN, MacCracken S, Mastrandrea PR, White LL (Eds.), Climate Change 2014: Impacts, Adaptation, and Vulnerability. Part B: Regional Aspects. Contribution of Working Group II to the Fifth Assessment Report of the Intergovernmental Panel on Climate Change. Cambridge University Press, Cambridge, United Kingdom and New York, NY, USA, pp 1327-1370.

Hoogenboom G, Porter CH, Shelia V, Boote KJ, Singh U, White JW, Hunt LA, Ogoshi R, Lizaso JI, Koo J, Asseng S, Singels A, Moreno LP, Jones JW (2019) Decision Support System for Agrotechnology Transfer (DSSAT) Version 4.7.5 (https://DSSAT.net). DSSAT Foundation, Gainesville, Florida, USA.

IPCC (2014) Climate change 2014: synthesis report, In: Core Writing Team, Pachauri, R.K., Meyer, L.A. (Eds.), Contribution of Working Groups I, II and III to the Fifth Assessment Report of the Intergovernmental Panel on Climate Change. IPCC, Geneva, Switzerland.

Page 15/23 
IUSS Working Group WRB (2006) World reference base for soil resources 2006. World Soil Resources Reports No. 103. FAO, Rome.

Jafarnejad A, Sharif-Alhosseini M (2010) The responses of spring wheat cultivars to different planting dates in autumn in Neishabour. Iran J Field Crops Res 8: 983-994. (in Persian with English summary) https://dx.doi.org/10.22067/gsc.v8i6.8046.

Jamieson PD, Porter JR, Wilson DR (1991) A test of the computer-simulation model ARCHWHEAT1 on wheat crops grown in New Zealand. Field Crops Res 27: 337-350. https://doi.org/10.1016/0378-4290(91)90040-3

Jiang T, Dou Z, Liu J, Gao Y, Malone R W, Chen S, Feng H, Yu Q, Xue G, He J (2020) Simulating the influences of soil water stress on leaf expansion and senescence of winter wheat. Agric For Meteorol. 291: 108061. https://doi.org/10.1016/j.agrformet.2020.108061.

Jones JW, Hoogenboom G, Porter CH, Boote KJ, Batchelor WD, Hunt L, Wilkens PW, Singh U, Gijsman AJ, Ritchie JT (2003) The DSSAT cropping system model. Eur J Agron 18: 235-265. https://doi.org/10.1016/S1161-0301(02)00107-7.

Jones PG, Thornton PK (2013) Generating downscaled weather data from a suite of climate models for agricultural modelling applications. Agric Syst 114: 15. https://doi.org/10.1016/j.agsy.2012.08.002.

Karandish F, Kalanaki M, Saberali SF (2017) Projected impacts of global warming on cropping calendar and water requirement of maize in a humid climate. Arch Agron Soil Sci 63: 1-13. https://doi.org/10.1080/03650340.2016.1177176.

Keating BA, Meinke H, Probert ME, Huth NI, Hills IG (2001) NWheat: Documentation and Performance of a Wheat Module for APSIM. Tropical Agriculture Technical Memorandum 9, CSIRO, Indooroopilly, Australia. https://doi.org/10.25919/g58j-5t69.

Kothari K, Ale S, Attia A, Rajan N, Xue Q, Munster CL (2019) Potential climate change adaptation strategies for winter wheat production in the Texas High Plains. Agric Water Manag 225: 105764. https://doi.org/10.1016/j.agwat.2019.105764.

Kothari K, Ale S, Attia A, Rajan N, Xue Q, Munster C.L (2019) Potential climate change adaptation strategies for winter wheat production in the Texas High Plains. Agric Water Manag 225: 105764. https://doi.org/10.1016/j.agwat.2019.105764.

Liu B, Liu L, Asseng S, Zou X, Li J, Cao W, Zhu Y (2016) Modelling the effects of heat stress on post-heading durations in wheat: A comparison of temperature response routines. Agric For Meteorol 222: 45-58. https://doi.org/10.1016/j.agrformet.2016.03.006.

Madani K, AghaKouchak A, Mirchi A (2016) Iran's socioeconomic drought: Challenges of a water-bankrupt nation. Iran Stud 49: 9971016. https://doi.org/10.1080/00210862.2016.1259286.

Malash NM, Flowers TJ, Ragab R (2008) Effect of irrigation methods, management and salinity of irrigation water on tomato yield, soil moisture and salinity distribution. Irrig Sci 26: 313-323. https://doi.org/10.1007/s00271-007-0095-7.

Mesgaran MB, Madani K, Hashemi H, Azadi P (2017) Iran's Land Suitability for Agriculture. Sci Rep 7: 7670. https://doi.org/10.1038/s41598-017-08066-y.

Mishra A, Singh R, Raghuwanshi NS, Chatterjee C, Froebrich J (2013) Spatial variability of climate change impacts on yield of rice and wheat in the Indian Ganga Basin. Sci. Total Environ 469: 132-138. https://doi.org/10.1016/j.scitotenv.2013.05.080.

NOAA National Centers for Environmental Information, State of the Climate: Global Climate Report for Annual 2020, published online January 2021. https://www.ncdc.noaa.gov/sotc/global/202013. Accessed 18 November 2021.

Nouri M, Homaee M, Bannayan M, Hoogenboom G (2017) Towards shifting planting date as an adaptation practice for rainfed wheat response to climate change. Agric Water Manag 186: 108-119. https://doi.org/10.1016/j.agwat.2017.03.004.

Reynolds MP, Saint Pierre C, Saad ASI, Vargas M, Condon A.G (2007) Evaluating potential genetic gains in wheat associated with stress-adaptive trait expression in elite genetic resources under drought and heat stress. Crop Sci 47: 172-189. https://doi.org/10.2135/cropsci2007.10.0022IPBS.

Robertson, GW (1968) A biometeorological time scale for a cereal crop involving day and night temperature and photoperiod. Int J Biometeorol 12: 191223. https://doi.org/10.1007/BF01553422.

Saberali, SF, Nastari-Nasrabadi H, Dostkhah-Ahmadi M (2019) Investigating the Impact of Climate Change on Irrigated Wheat Production under Torbat-e Jam Region for the Near Future. Iran J Field Crops Res. 17: 169-186. (in Persian with English summary) https://doi.org/10.22067/gsc.v17i1.74284.

Salem BB (1989) Arid Zone Forestry: A Guide for Field Technicians. Food and Agriculture Organization (FAO) Conservation Guide 20. Rome, Italy.

Shewry PR (2009) Wheat. J Exper Bot 60: 1537-1553. https://doi.org/10.1093/jxb/erp058.

Shirmohammadi-Aliakbarkhani Z., Saberali SF (2020) Evaluating of eight evapotranspiration estimation methods in arid regions of Iran. Agric Water Manag 239: 106243. https://doi.org/10.1016/j.agwat.2020.106243

Shojaee T, Fallah-Ghalhari G (2017) Variations trend of climate parameters affecting on grape growth (Case study: Khorasan Razavi Province). Nat. Clim. Chang. 3: 45-58. https://doi.org/10.22059/jnec.2017.234944.68. 
Smit B, Skinner MW (2002) Adaptation options in agriculture to climate change: A typology. Mitig Adapt Strateg Glob Change 7: 85-

114. https://doi.org/10.1023/A:1015862228270.

Sommer R, Glazirina M, Yuldashev T, Otarov A, Ibraeva M, Martynova L, Bekenov M, Kholov B, Ibragimov N, Kobilov R, Karaev R, Sultonov M, Khasanova F, Esanbekov M, Mavlyanov D, Isaev F, Abdurahimov A, Ikramov R, Shezdyukova L, de Pauw E (2013) Impact of climate change on wheat productivity in Central Asia. Agric Ecosyst Environ 178: 78-99. https://doi.org/10.1016/j.agee.2013.06.011.

Vaghefi SA, Keykhai M, Jahanbakhshi F, Sheikholeslami J, Ahmadi A, Yang H, Abbaspour KC (2019) The future of extreme climate in Iran. Sci Rep 9: 1464. https://doi.org/10.1038/s41598-018-38071-8.

Valizadeh J, Ziaei SM, Mazloumzadeh SM (2014) Assessing climate change impacts on wheat production (a case study). Journal of the Saudi Society of Agricultural Sciences, 13: 107-115. https://doi.org/10.1016/j.jssas.2013.02.002.

White JW, Kimball BA, Wall GW, Ottman MJ (2012) Field Crops Research Cardinal temperatures for wheat leaf appearance as assessed from varied sowing dates and infrared warming. Field Crops Res 137: 213-220. https://doi.org/10.1016/j.fcr.2012.08.013.

Yin XY, Guo W, Spiertz JH (2009) A quantitative approach to characterize sink-source relationships during grain filling in contrasting wheat genotypes. Field Crops Res 114: 119-126. https://doi.org/10.1016/j.fcr.2009.07.013.

Zamanirad M, Sedghi H, Sarraf A, Saremi A, Rezaee P (2018) Potential impacts of climate change on groundwater levels on the Kerdi-Shirazi plain, Iran. Environ Earth Sci 77: 1-10. https://doi.org/10.1007/s12665-018-7585-1.

\section{Figures}

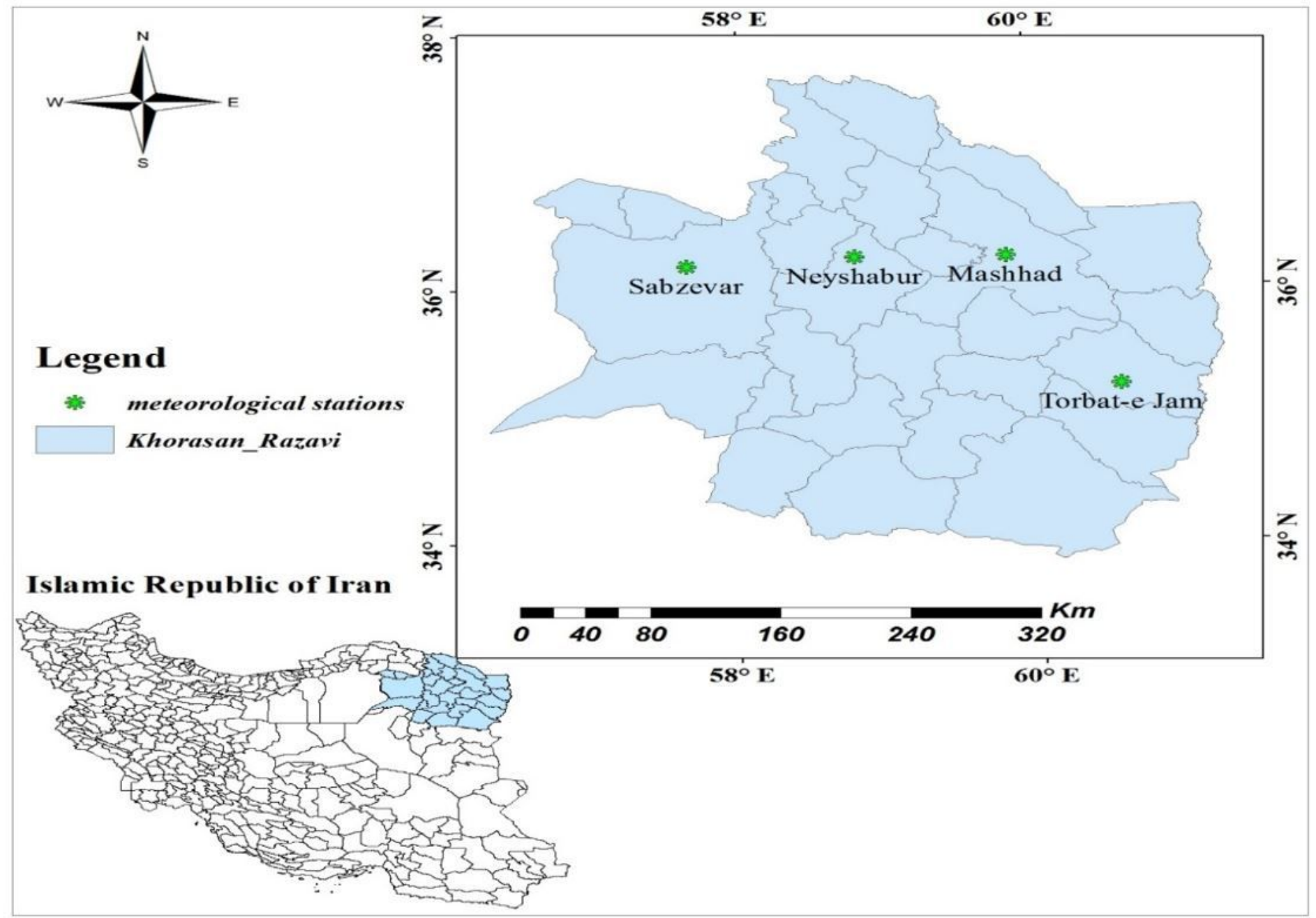

Figure 1

Locations of the four major wheat growing districts in Khorasan Razavi province. 

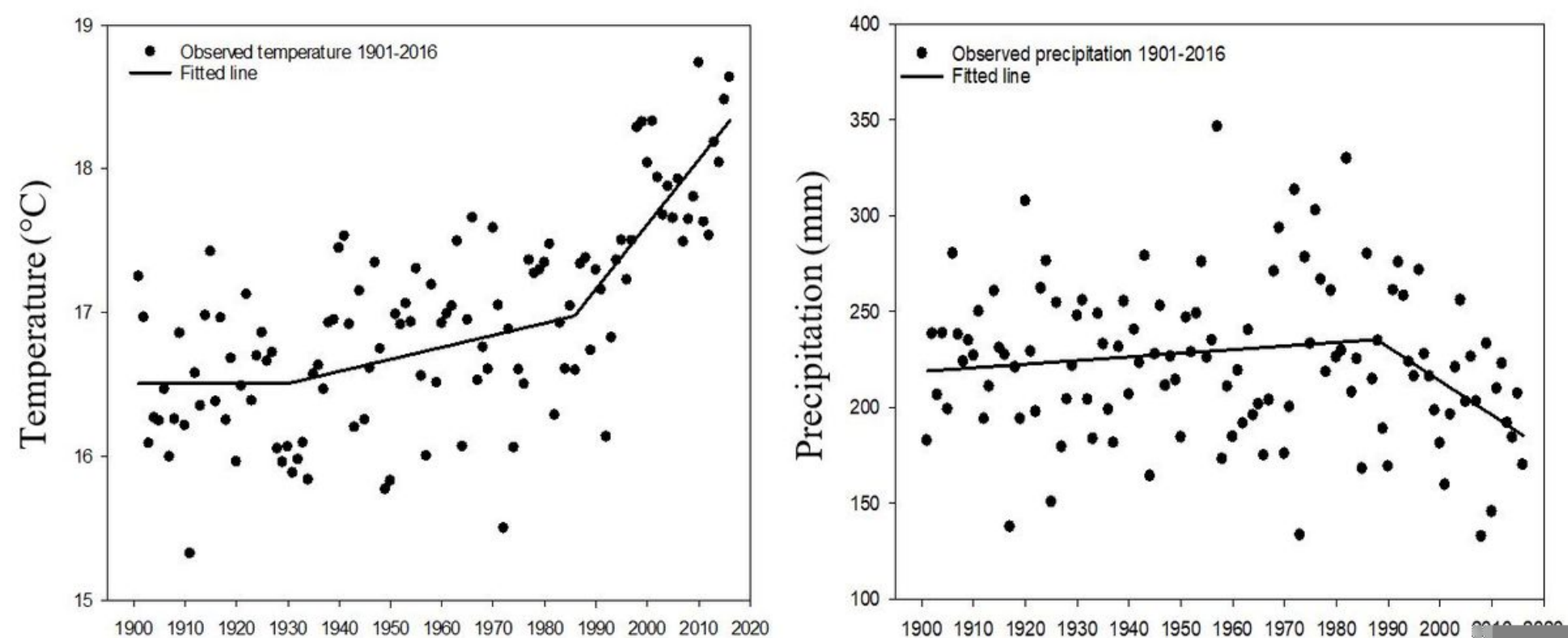

Figure 2

The mean annual temperature and total annual precipitation over the period of 1901-2016 in Iran. The data was obtained from the Climate Data API of the World Bank.

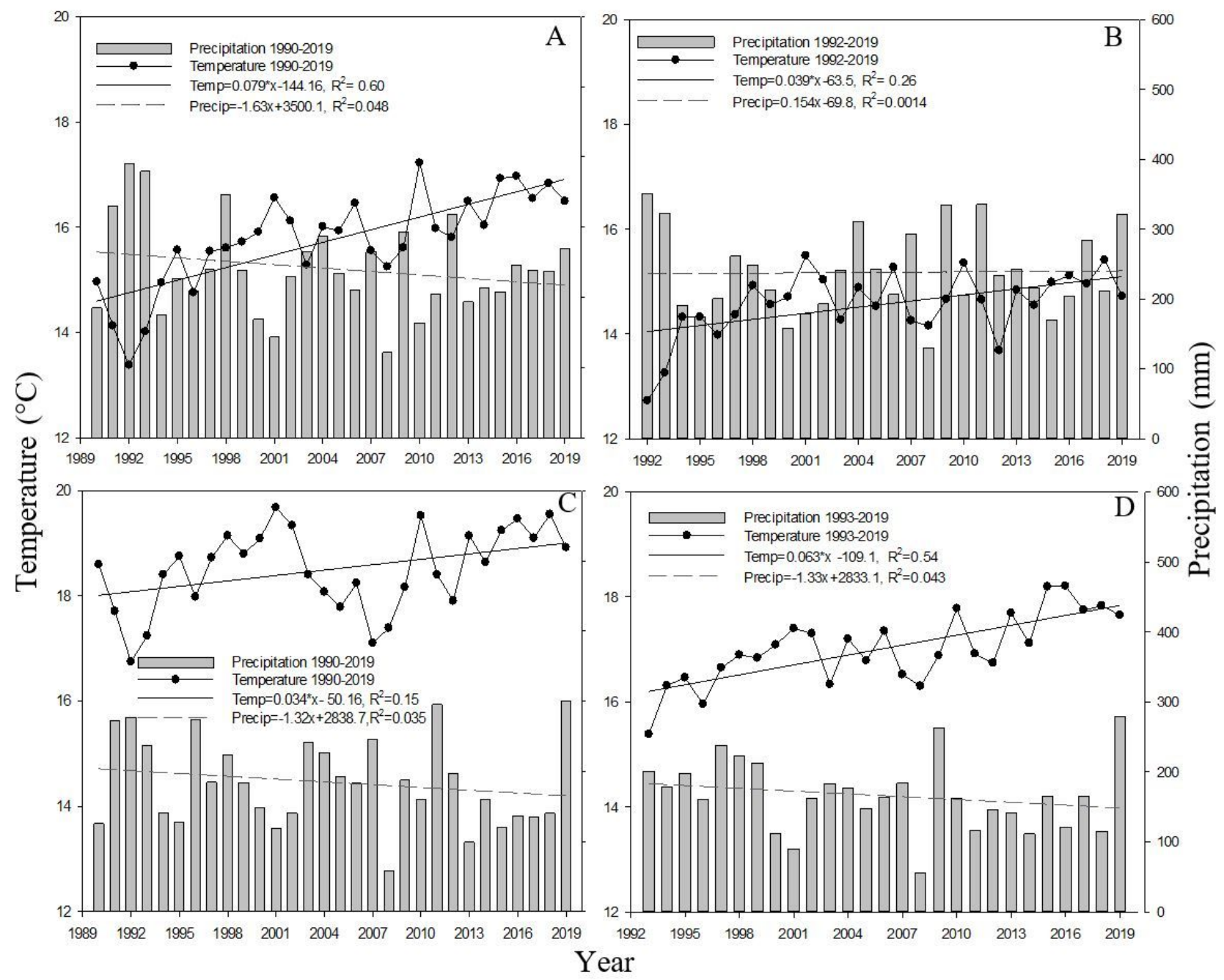


Figure 3

The mean annual temperature, annual precipitation and their changing trends over last 27-30 years in (A) Mashhad, (B) Neyshabour, (C) Sabzevar, and (D) Torbat-e Jam.

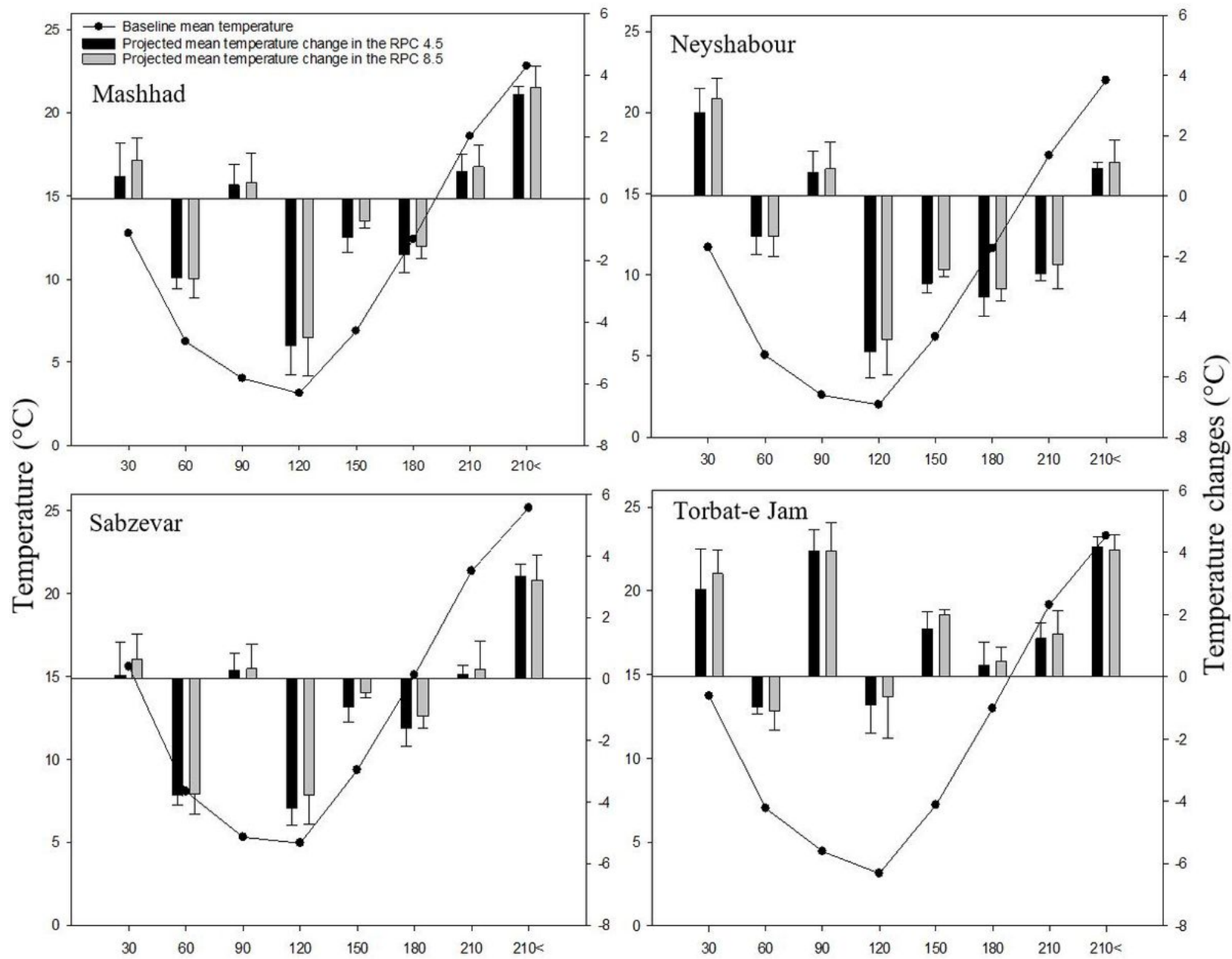

Days after planting

Figure 4

The baseline mean temperature and the projected change in mean temperature during the winter wheat growing season under RCP4.5 and RCP8.5 climate scenarios. The error bars represent standard deviation, which is indicative of the uncertainty in the climate projections. 

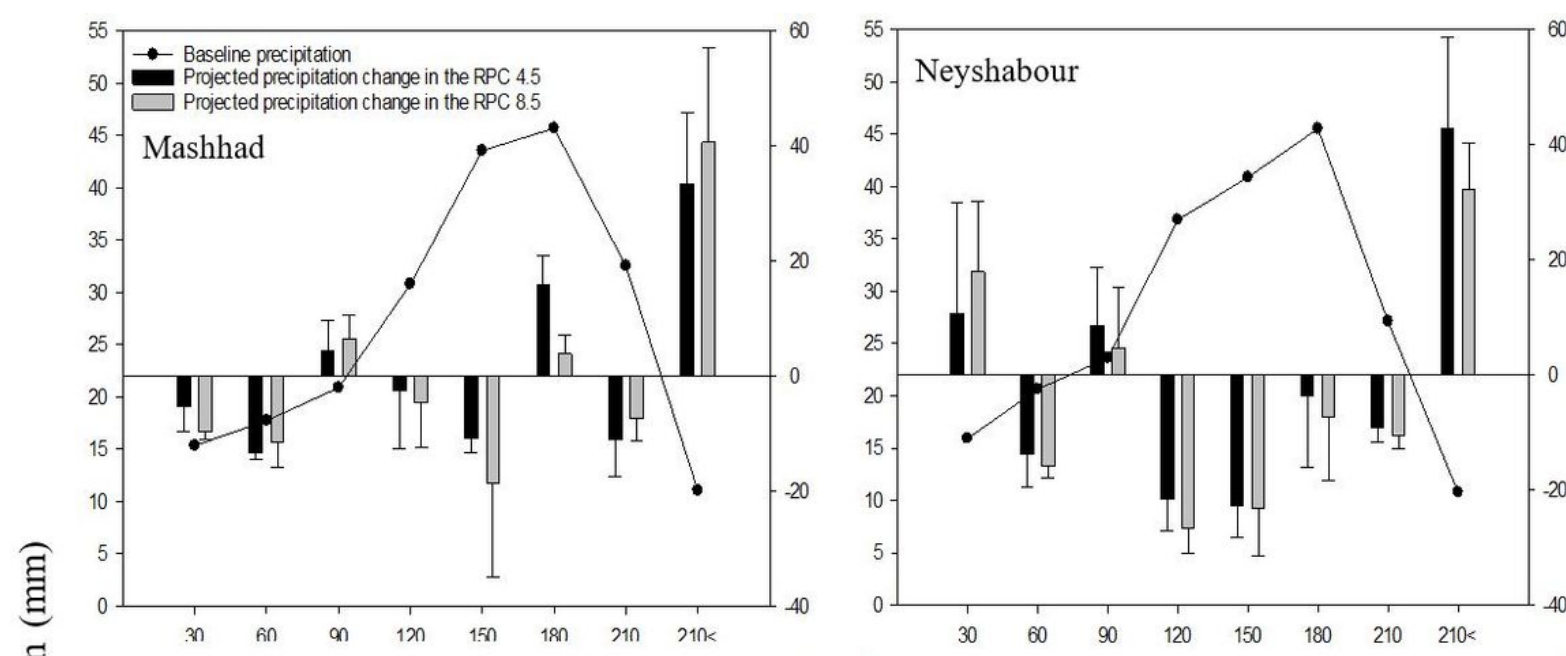

60
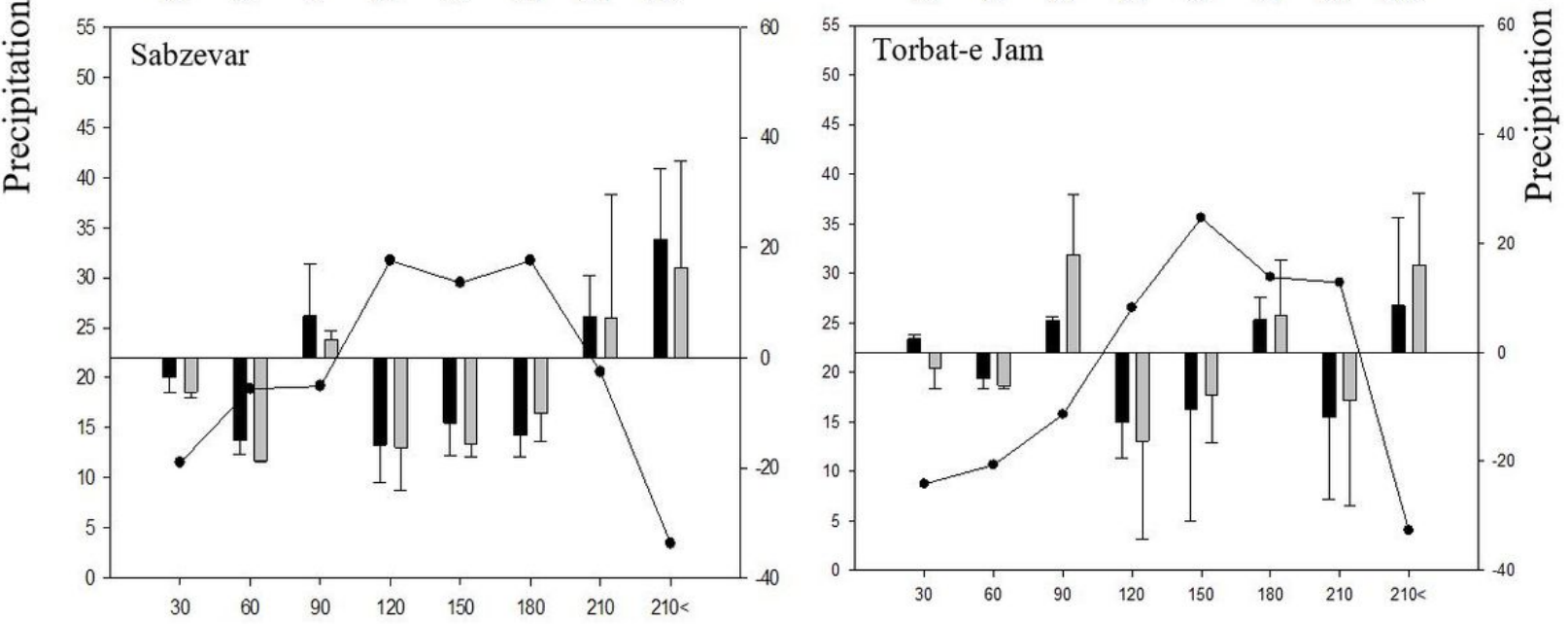

Days after planting

\section{Figure 5}

The baseline precipitation rates and the projected changes in precipitation during the winter wheat growing season under RCP4.5 and RCP8 climate scenarios. The error bars represent standard deviation, which is indicative of the uncertainty in the climate projections. 

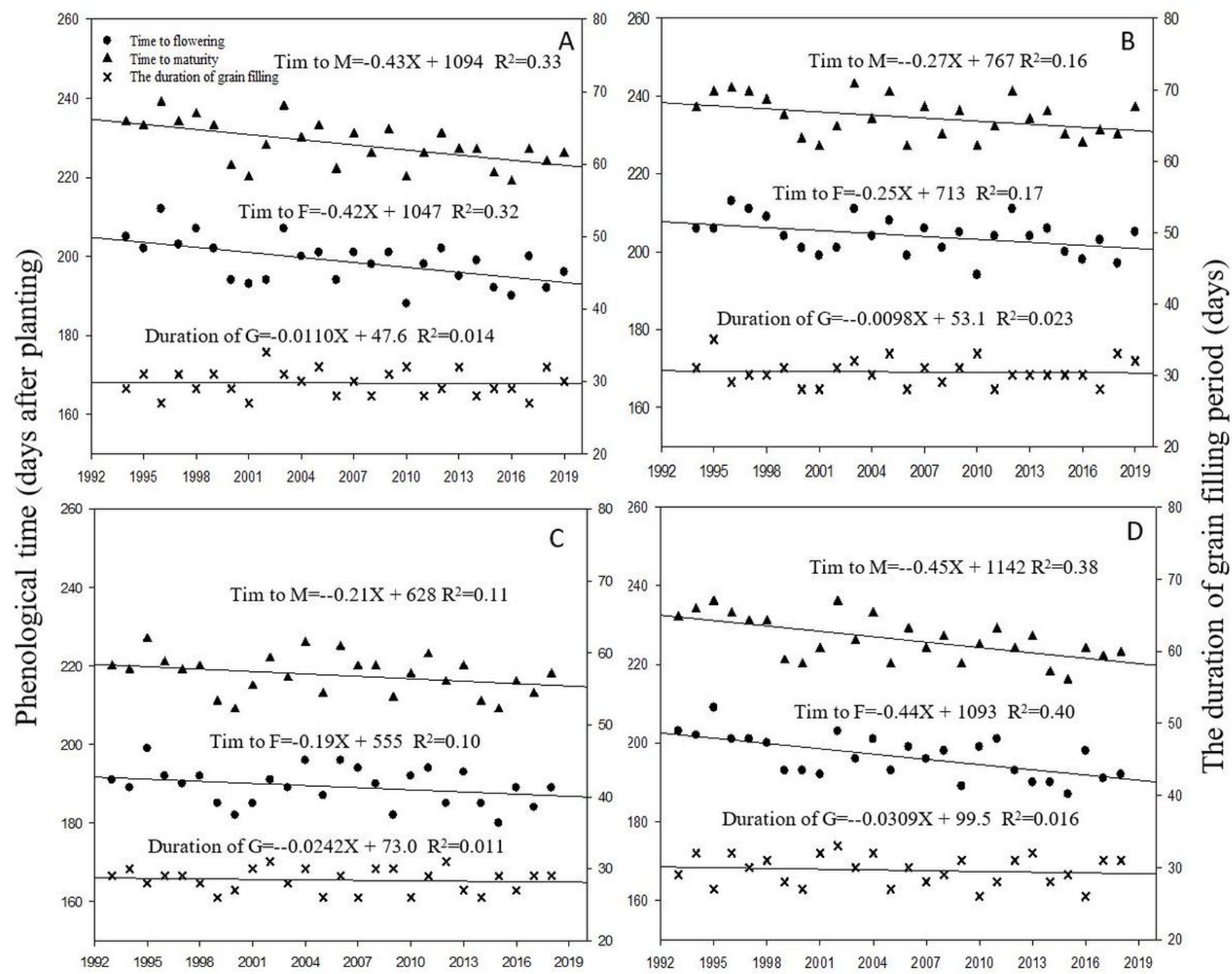

The baseline study period (years)

\section{Figure 6}

The trends of simulated days to flowering and maturity, and duration of grain filling period over the baseline period (1993-2019) in (A) Mashhad, (B) Neyshabour, (C) Sabzevar, and(D) Torbat-e Jam. 

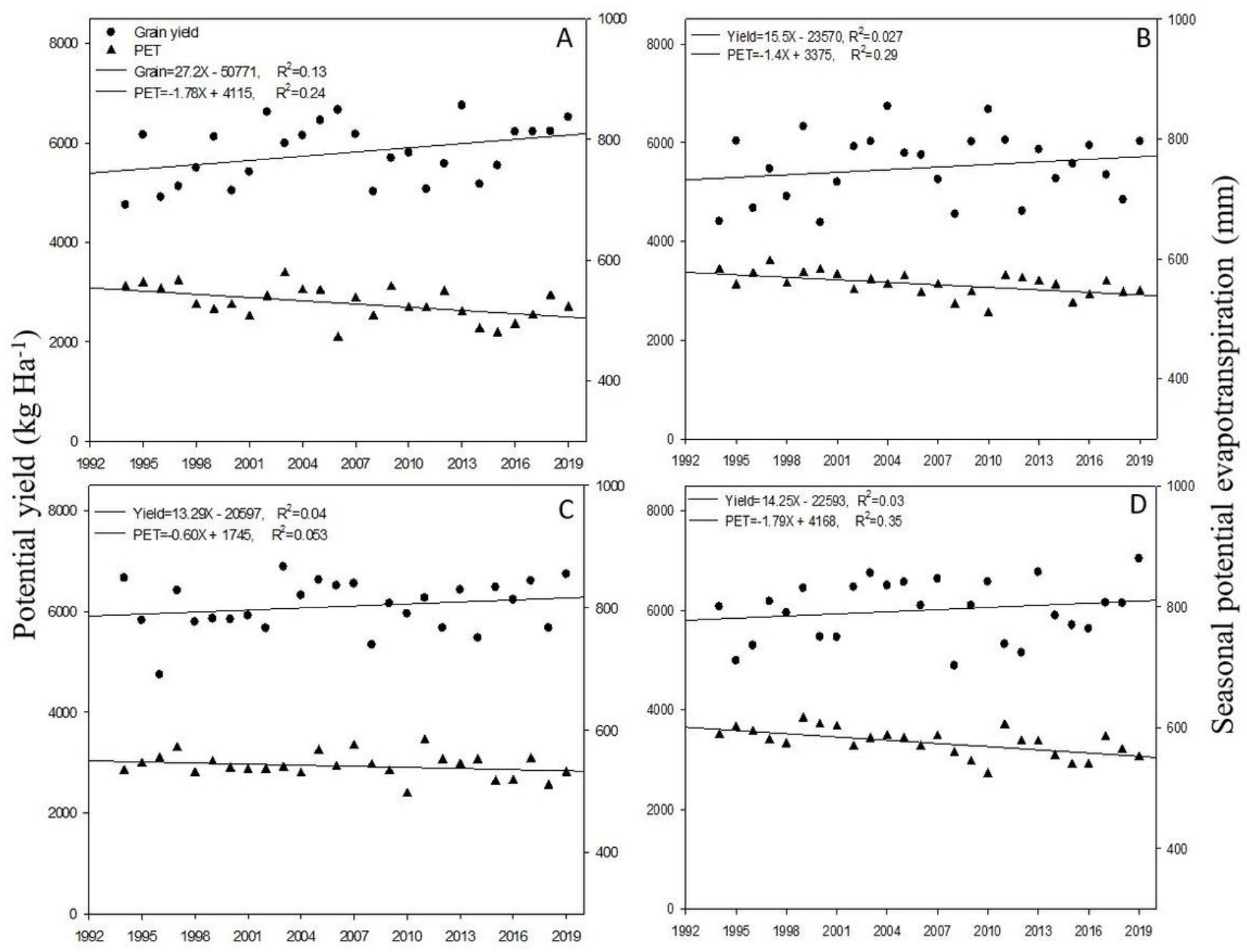

The baseline study period (years)

\section{Figure 7}

The temporal trends of simulated potential yield and growing season evapotranspiration of winter wheat over the baseline period (1993-2019) in (A) Mashhad,

(B) Neyshabour, (C) Sabzevar, and(D) Torbat-e Jam. 


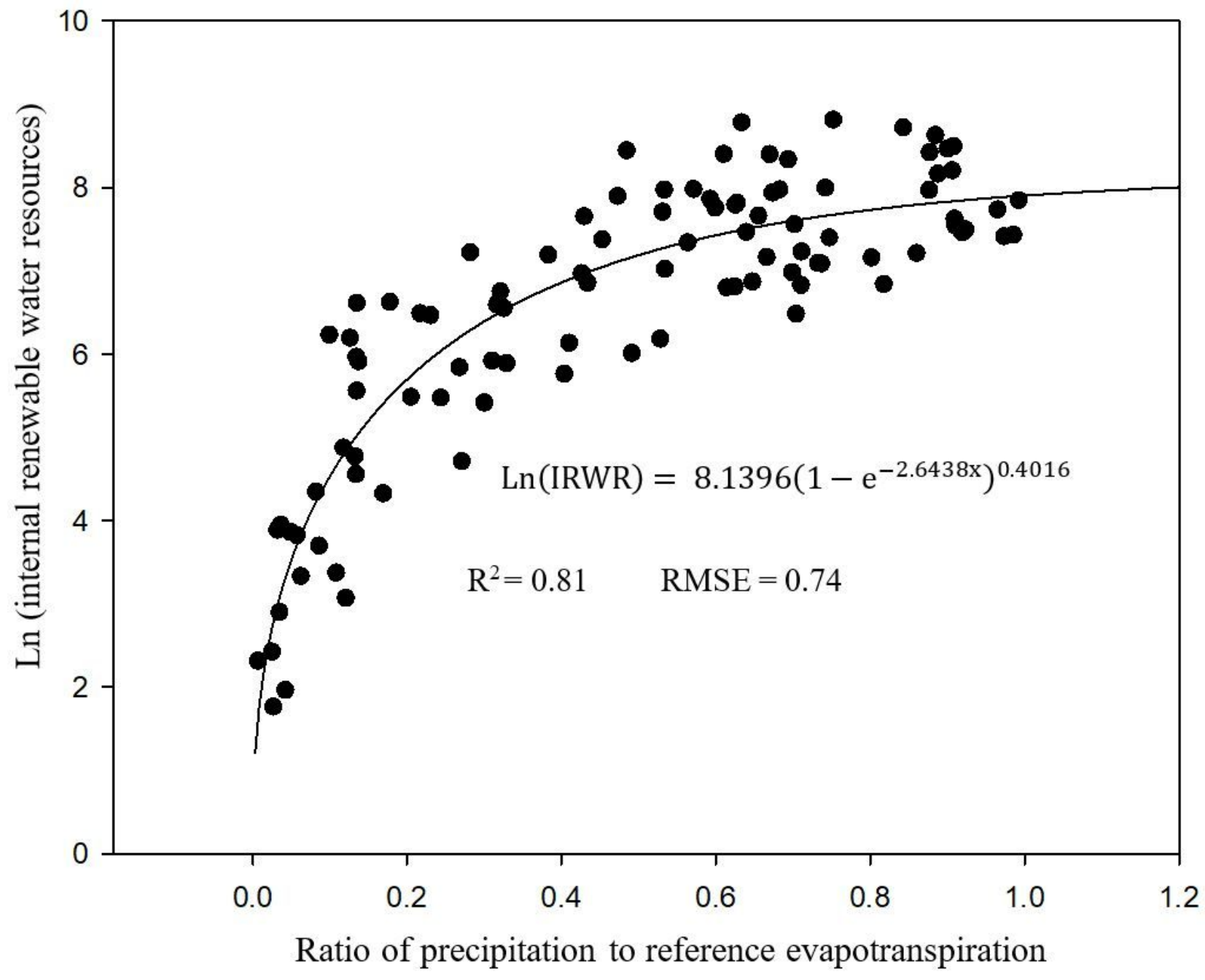

Figure 8

The relationship between the natural logarithm of internal renewable water resources (IRWR) and the ratio of precipitation (P) to reference evapotranspiration (ETo) for different countries around the world. The data for the IRWR, annual ETo and annual P for different countries was obtained from FAO AQUASTAT database. 